\title{
The impact of astrophysical dust grains on the confinement of cosmic rays
}

\author{
Jonathan Squire ${ }^{\oplus},{ }^{1 \star}$ Philip F. Hopkins ${ }^{\oplus},{ }^{2}$ Eliot Quataert ${ }^{3,4}$ and Philipp Kempski ${ }^{3}$ \\ ${ }^{1}$ Physics Department, University of Otago, Dunedin 9010, New Zealand \\ ${ }^{2}$ TAPIR, Mailcode 350-17, California Institute of Technology, Pasadena, CA 91125, USA \\ ${ }^{3}$ Astronomy Department and Theoretical Astrophysics Center, University of California, Berkeley, CA 94720, USA \\ ${ }^{4}$ Department of Astrophysical Sciences, Princeton University, Princeton, NJ 08544, USA
}

Accepted 2021 January 18. Received 2021 January 18; in original form 2020 November 3

\begin{abstract}
We argue that charged dust grains could significantly impact the confinement and transport of galactic cosmic rays. For sub-GeV to $\sim 10^{3} \mathrm{GeV}$ cosmic rays, small-scale parallel Alfvén waves, which isotropize cosmic rays through gyro-resonant interactions, are also gyro-resonant with charged grains. If the dust is nearly stationary, as in the bulk of the interstellar medium, Alfvén waves are damped by dust. This will reduce the amplitude of Alfvén waves produced by the cosmic rays through the streaming instability, thus enhancing cosmic ray transport. In well-ionized regions, the dust damping rate is larger by a factor of $\sim 10$ than other mechanisms that damp parallel Alfvén waves at the scales relevant for $\sim \mathrm{GeV}$ cosmic rays, suggesting that dust could play a key role in regulating cosmic ray transport. In astrophysical situations in which the dust moves through the gas with super-Alfvénic velocities, Alfvén waves are rendered unstable, which could directly scatter cosmic rays. This interaction has the potential to create a strong feedback mechanism where dust, driven through the gas by radiation pressure, then strongly enhances the confinement of cosmic rays, increasing their capacity to drive outflows. This mechanism may act in the circumgalactic medium around star-forming galaxies and active galactic nuclei.
\end{abstract}

Key words: instabilities-plasmas-cosmic rays-galaxies: evolution.

\section{INTRODUCTION}

The mechanisms that enable and constrain the propagation and generation of cosmic rays (CRs) in galaxies remain only partially understood (Bell 2013; Amato \& Blasi 2018). While it is well accepted that, in order to explain their observed isotropy, CRs must scatter from small-scale irregularities of the magnetic field, the origin and properties of these irregularities is uncertain. Key globally averaged quantities, such as the average CR escape time, can be constrained by local observations of the CR spectrum and composition (e.g. Strong \& Moskalenko 1998; Ahn et al. 2010; Adriani et al. 2011; Evoli et al. 2017). In order to better understand these observations, it is desirable to improve our theoretical grasp of the physics governing particle transport across a range of energies and under different galactic conditions.

The problem is particularly acute for the relatively low energy $\sim \mathrm{GeV}$ protons that dominate the $\mathrm{CR}$ energy density, for two reasons. First, such CRs are arguably the most difficult to understand theoretically: they interact with extremely small $\lesssim \mathrm{AU}$ scales, and precisely because they dominate the energy density - their transport is likely controlled by magnetic structures that they themselves excite (making the relevant processes highly non-linear). Secondly, and again because they dominate the energy density, $\sim \mathrm{GeV}$ CRs are thought to be important for a variety of global processes in galactic astrophysics, such as launching large-scale outflows and modifying the phase structure of the background gas (e.g. Pfrommer et al.

^E-mail: jonathan.squire@otago.ac.nz
2017; Butsky \& Quinn 2018; Ji et al. 2020; Bustard \& Zweibel 2020; Su et al. 2020; Hopkins et al. 2021a, and references therein). This makes understanding the details of CR transport and scattering, including the dependence on local properties of the background gas (e.g. temperature, density, magnetic field strength, and ionization fraction), particularly relevant for the $\sim \mathrm{GeV} \mathrm{CR}$ population. The recent study of Hopkins et al. (2021b) (hereafter H+20) has highlighted this uncertainty: using cosmological simulations and simple scaling models, they found that none of the popular theoretical models of $\sim \mathrm{GeV}$ CR propagation could satisfactorily explain local grammage measurements or observations of gamma-ray emission from the haloes of other galaxies (Lacki et al. 2011; Griffin, Dai \& Thompson 2016; Lopez et al. 2018). We are thus motivated to examine possible omissions in current theories of CR transport.

In this work, we consider the impact of charged dust on cosmic ray transport. Although at first glance, dust-CR interactions may seem esoteric and unlikely to be significant (indeed, their direct Coulomb interactions are weak; Byleveld, Melrose \& Cram 1993), we find a surprisingly strong interaction that suggests dust-related effects could dominate CR transport in a number of astrophysically important regimes. The fundamental cause of the interaction is small-scale parallel Alfvén waves (AWs): such waves are a necessary ingredient in scattering CRs and thus key to their transport; but they also, by very similar mechanisms, interact strongly with charged dust. It further transpires that sub-AU-scale AWs, which are important to $\sim \mathrm{GeV} \mathrm{CR}$ transport, interact with a range of grain sizes and charges that are likely prevalent in the interstellar medium (ISM) and circumgalactic medium (CGM). We suggest two possible effects. The most important involves the CR 'self-confinement' scenario, 
whereby CRs excite small-scale AWs through the 'streaming instability,' which subsequently grow in time, scattering CRs and reducing their transport (Wentzel (Kulsrud \& Pearce 1969; Wentzel $1969,1974)$. In this scenario, dust acts to damp the small-scale parallel AWs excited by CRs (Cramer, Verheest \& Vladimirov 2002), reducing the effectiveness of scattering and enhancing $\mathrm{CR}$ transport. We find that dust-damping rates are large enough, even given the low mass fraction of dust in the ISM, for the effect to dominate over all other AW-damping mechanisms in well-ionized gas. The second possible effect involves dust that is moving through the gas with super-Alfvénic velocities due to radiation pressure (or some other force that affects the gas and dust differently). As studied in detail in Hopkins \& Squire (2018) (hereafter HS18), AWs are unstable in this situation with a similar mechanism to the CR streaming instability; these AWs may then scatter CRs and enhance their confinement. Although the effect is more difficult to assess in detail than the AW damping, because of significant uncertainties in wave saturation physics and dust astrophysics, we speculate that it has the potential to provide a rather strong feedback mechanism by enhancing the coupling of CRs to CGM gas.

We start the paper in Section 2 with an overview of the theory of CR transport and AWs, covered in sufficient detail to allow later discussion of the effect of dust. Section 3, with further details in Appendix A, covers the calculation of the damping or growth rate of AWs in the presence of a spectrum of grain sizes. We then consider the astrophysical implications of these results in Section 4, covering CR self-confinement in the ISM in Section 4.2, and speculating on dust-enhanced CR confinement in the CGM in Section 4.3. We conclude in Section 5. A list of symbols used throughout the text is given in Table 1.

\section{COSMIC RAY SCATTERING AND SELF - CONFINEMENT}

In this section, we outline the basic theory of Cosmic Ray (CR) scattering, briefly reviewing the relevant physics. We also point out some interesting features related to wave polarization that are specific to Alfvén wave interaction with dust, exploring how this could affect our later estimates of CR transport (this discussion, in Section 2.1, is unimportant to the overall narrative and may be skimmed without causing problems later in the text).

CR scattering is thought to occur primarily through their interaction with parallel Alfvén waves (AWs) with a wavelength that is resonant with the distance they stream along the magnetic field in one gyro-orbit (Jokipii 1966): $k_{\text {res }} \xi v \approx \pm \Omega_{c}$, where $k_{\text {res }}$ is the resonant wavenumber of the $\mathrm{AW}, \xi v=v_{\|}$is the velocity along the field line of a particle with pitch angle $\xi=v_{\|} / v$, and $\Omega_{c}$ is the particle's gyrofrequency. Neglecting the $\xi$ dependence of the resonant condition and the distribution of thermal velocities, the condition for highly relativistic protons simplifies to

$k_{\text {res }}^{-1} \approx r_{L} \approx 3.3 \times 10^{12} \mathrm{~cm}\left(\frac{R^{\mathrm{CR}}}{\mathrm{GV}}\right)\left(\frac{B}{1 \mu \mathrm{G}}\right)^{-1}$,

where $B$ is the magnetic field strength, $R^{\mathrm{CR}}$ is the $\mathrm{CR}$ rigidity, and equation (1) applies for $R^{\mathrm{CR}} \gtrsim 1 \mathrm{GV}$. For a given relative power $\delta \boldsymbol{B}^{2}\left(k_{\|}\right) / B^{2}$ in magnetic field fluctuations at parallel scale $k_{\|}$, the scattering frequency is $v_{c} \sim \Omega_{c} \delta \boldsymbol{B}^{2}\left(k_{\|}\right) / B^{2}$. The scattering brings the CRs back towards isotropy in the frame of the waves, thus reducing the transport of CR energy. With sufficiently efficient scattering - i.e. with sufficient power in $r_{L}$-scale parallel waves - the CRs behave like a fluid that drifts at the speed of the scatterers with respect to the gas (Skilling 1975a; McKenzie \& Voelk 1982; Thomas
Table 1. Important symbols used throughout this article. In the linear calculations, a subscript ' $i$ " indicates the quantity for grains of species/size $i$. In astrophysical estimates, we use a numerical subscript to denote order of magnitude in cgs units, $F_{x}=F / 10^{x}$; so, e.g. $T_{4}=$ $T /\left(10^{4} \mathrm{~K}\right), n_{0}=n /\left(1 \mathrm{~cm}^{-3}\right)$, and $a_{-5}=a_{d} /(0.1 \mu \mathrm{m})$.

\begin{tabular}{|c|c|}
\hline Symbol & Description and/or definition \\
\hline$r_{L}$ & CR gyro-radius \\
\hline$\xi=v_{\|} / v$ & CR pitch angle ${ }^{a}$ \\
\hline$v_{c}$ & CR scattering frequency \\
\hline$\kappa_{\|}, \tilde{v}_{\mathrm{st}}$ & CR diffusivity, 'effective' CR streaming speed \\
\hline$\rho, \boldsymbol{u}$ & Gas mass density, velocity \\
\hline$B, v_{A}$ & Magnetic-field strength, Alfvén speed \\
\hline$T, n$ & Gas temperature, number density \\
\hline$\beta=8 \pi n k_{B} T / B^{2}$ & Ratio of thermal to magnetic pressure \\
\hline$\rho_{d}, \boldsymbol{v}$ & Dust mass density, bulk velocity \\
\hline$\mu_{0}=\left\langle\rho_{d}\right\rangle /\langle\rho\rangle$ & Total dust-to-gas mass ratio \\
\hline$a_{d}$ & Dust-grain size \\
\hline$a_{d, \min }, a_{d, \max }$ & Minimum/maximum grain size in distribution \\
\hline$\xi_{\mu}$ & Slope of dust mass distribution \\
\hline$t_{s}\left(a_{d}\right)$ & Grain stopping time due to dust-gas drag \\
\hline$t_{L}\left(a_{d}\right)$ & Grain Larmor time \\
\hline$U_{d}\left(a_{d}\right)$ & Grain electrostatic potential \\
\hline $\bar{U}, \bar{U}_{0}$ & Normalized $U_{d}$ (collisional or photoelectric) \\
\hline$a^{\text {ext }}$ & Grain acceleration from radiation pressure \\
\hline$w_{s}$ & Equilibrium grain drift velocity in gas frame \\
\hline $\mathcal{W}_{s}=w_{s} / v_{A}-1$ & Alfvénically normalized grain drift velocity \\
\hline$\xi_{t_{L}}, \xi_{\mathcal{W}_{s}}$ & Scaling of $t_{L}, \mathcal{W}_{s}$ with grain size \\
\hline$k$ & (Parallel) wavenumber of instability \\
\hline$\omega_{A}=k v_{A}$ & Alfvén frequency \\
\hline$\Gamma$ & Instability growth or damping rate \\
\hline$k_{a_{d, \min }}, k_{a_{d, \max }}$ & Maximum, minimum unstable wavenumber \\
\hline
\end{tabular}

\& Pfrommer 2019). With less scattering, however, CRs are not efficiently isotropized, leading to significantly more energy transport. Thus, understanding the power in $r_{L}$-scale parallel fluctuations is crucial to understanding CR transport. In the ISM, the $r_{L}$ scale is tiny ( $\lesssim \mathrm{AU}$ ) for the $\sim \mathrm{GeV}$ CRs that dominate the energy density, and although interstellar turbulence is expected to put significant power into perpendicular Alfvénic fluctuations on such scales, perpendicular fluctuations scatter CRs very inefficiently and likely cannot account for the observed isotropy of CRs (Chandran 2000; Yan \& Lazarian 2002; H+20). This suggests that other sources of small-scale parallel AWs are needed. Note that CRs can, in principle, scatter from non-Alfvénic fluctuations also, but such fluctuations are strongly damped at $r_{L}$ scales and there remains debate as to whether they can play a significant role (Yan \& Lazarian 2004, 2008; H+20).

Given this apparent inability of turbulence to provide sufficient CR scattering, the most commonly accepted source of $r_{L}$-scale fluctuations is the CRs themselves. In the standard 'self-confinement' picture of CR transport (Kulsrud \& Pearce 1969; Wentzel 1969; Skilling 1971), streaming CRs generate parallel AWs due to the 'streaming instability' whenever their bulk drift velocity exceeds the local Alfvén speed of the background plasma. The waves, with wavelengths that automatically match the CR gyro-radii, then efficiently scatter particles, bringing the CR bulk drift speed back towards the Alfvén speed. If undamped, it is expected that such AWs would grow to large amplitudes, causing efficient scattering of CRs and bringing their drift back to nearly exactly the Alfvén speed (Skilling 
1975a, b). In contrast, if there exists an efficient mechanism to damp parallel AWs, the CR-AW system is expected to come to a local equilibrium where the growth rate of AWs due to the CR streaming is balanced by damping, implying that the CR energy density can be transported at speeds significantly faster than the Alfvén speed. Thus, in the self-confinement paradigm, an understanding of parallel AW damping is crucial to understanding CR transport: more efficient damping leads to faster CR transport. Current understanding suggests that ion-neutral viscosity (Kulsrud \& Pearce 1969), damping from the interaction of parallel Alfvén waves with turbulence (Farmer \& Goldreich 2004; Zweibel 2017), and non-linear Landau damping (Lee \& Völk 1973; Cesarsky \& Kulsrud 1981; Völk \& Cesarsky 1982), are the most important physical processes. In predominantly neutral gas (e.g. cold clouds, the warm neutral medium), ion-neutral damping is highly dominant and leads to rapid transport of CRs. However, as the ionization fraction increases, ion-neutral damping rates quickly drop to zero. Through galaxy-scale simulations and simple analytic arguments, $\mathrm{H}+20$ argued that it is transport through well-ionized gas that dominates the global confinement properties, while fast damping rates (fast transport) in predominantly neutral regions make little difference (see also Farber et al. 2018). Further, $\mathrm{H}+20$ found that current self-confinement transport models predict too much CR confinement in ionized gas to match observations.

We are thus left with two possible roles for dust in regulating CR transport. In the first - 'external confinement' - drifting dust may directly excite small-scale parallel AWs due to instability. Such waves, if they can reach large amplitudes, will scatter CRs directly, thus increasing their confinement. In the second - 'self-confinement' - near-stationary dust will act to damp small-scale AWs excited by the CRs themselves, thus decreasing their confinement.

\subsection{Polarization}

An interesting feature of dust-AW interaction, which may have consequences for both external confinement and self-confinement of CRs, is the dependence on wave polarization. Specifically, CRs with a given sign of $v_{\|}$interact only with one AW polarization: righthanded waves propagating parallel (or left-handed waves propagating antiparallel) to the magnetic field scatter only $v_{\|}>0$ positively charged CRs, while the opposite is true for $v_{\|}<0$ CRs. The CR streaming instability excites nearly linearly polarized AWs because the populations of $v_{\|}<0$ and $v_{\|}>0$ particles are nearly the same (Kulsrud \& Pearce 1969; Bai et al. 2019); however, only waves that propagate in the CR-drift direction are unstable. For similar reasons, AW damping or instability due to dust is also dependent on wave polarization, in contrast to the other wave damping mechanisms (e.g. turbulence damping) mentioned above. In particular (see Section 3 below), for waves propagating along the magnetic field direction, negatively (positively) charged stationary dust damps only righthanded (left-handed) waves; vice-versa for waves propagating in the antifield direction. Similarly, dust drifting super-Alfvénically in the magnetic field direction excites left-handed waves if negatively charged, or right-hand waves if positively charged, and in both cases only waves propagating in the same direction as the dust are unstable. In this section, we consider how damping or growth of just one polarization of AW would affect CR self-confinement and scattering. We wish to understand how to appropriately compare AW damping or growth rates from dust with those due to other mechanisms.

First consider the self-confinement scenario, with dust providing the dominant damping mechanism for nearly linearly polarized AWs excited by the CR streaming instability. As noted above, dust (if negatively charged) will damp only the right-handed (forward propa- gating) waves, thus leaving left-handed waves to grow freely to larger amplitudes. There will thus be efficient scattering of $v_{\|}<0$ particles, which will tend to make the $\mathrm{CR}$ distribution function independent of $\xi$ for $v_{\|}<0$; but, the $v_{\|}>0$ part of the CR distribution function, which only interacts with the strongly damped waves, remains nearly unaffected. However, because the overall drift velocity of the CRs arises from the difference in the total population of $v_{\|}<0$ and $v_{\|}>$ 0 particles, flattening of the $v_{\|}<0$ part of the distribution in $\xi$ makes only a minor difference to the CR drift and energy transport, or to the streaming instability growth rate (the effect is nicely illustrated in Bai et al. 2019, fig. 9). Further, it seems likely that isotropization of particles across $\xi=0$ (often called the ' $90^{\circ}$ barrier') requires variation in $|\boldsymbol{B}|$, so would not be efficient in the presence of only one wave polarization (Felice \& Kulsrud 2001; Bai et al. 2019; Holcomb \& Spitkovsky 2019). Overall, this suggests that the effective transport will be approximately determined by the level of the lowest amplitude waves (right-handed in the discussion above), with little dependence on a possibly large-amplitude population of the other polarization. Thus, damping just one wave polarization through interaction with dust should have a similar effect on CR self-confinement to damping both polarizations, implying it is reasonable to compare dust AW damping rates directly to those from other sources that do not distinguish between polarizations (e.g. turbulence damping). Of course, there exist a variety of complexities in the above argument that make it highly uncertain; however, it seems likely that such questions may only be answered definitively with detailed simulations of the streaming instability, which are only recently becoming possible (Bai et al. 2019; Dubois et al. 2019; Haggerty \& Caprioli 2019; Holcomb \& Spitkovsky 2019; Weidl, Winske \& Niemann 2019).

From the above argument, we also see that Alfvén waves that are excited directly by drifting dust ('external confinement') may be rather inefficient at confining CRs. Before the instability saturates, and neglecting other sources of AWs including those excited by CRs, only one polarization of AW will be present. Such a spectrum of AWs will not significantly perturb the magnetic field strength and will only scatter CRs with one sign of $\xi$. Such a process can only limit the energy transport of CRs by a maximum factor of $\sim 2$. This effect was seen and diagnosed in detail in the CR streaming-instability simulations of Holcomb \& Spitkovsky (2019), where they noted that CRs with an initial drift velocity that is too large (approaching c) are inefficiently self-confined, because the wave scattering only isotropizes particles with one sign of $\xi$. However, the non-linear saturation of the dust instability presents significant uncertainties; if the saturation is quasi-turbulent in nature, as suggested by the simulations of Seligman, Hopkins \& Squire (2019), Hopkins et al. (2020c) (albeit in a different regimes), it seems implausible that only one wave polarization would be present in the saturated state. In that case, fluctuations induced by the dust could more efficiently isotropize CRs. There are clearly additional uncertainties, such as how different spatial regions behave when there is a large-scale gradient of CRs, and detailed simulations of both the saturation of the dust instability and the CR scattering are needed for better understanding of the system. Overall, however, it seems reasonable to surmise that CR confinement from small-scale AWs excited by dust could be less efficient than suggested by estimates of $\delta \boldsymbol{B}^{2}\left(k_{\|}\right) / B^{2}$ alone.

\section{THE INTERACTION OF HIGHLY CHARGED DUST WITH ALFVÉN WAVES}

The goal of this section is to compute the damping or growth rate of parallel shear-Alfvén waves in the presence of a wide spectrum of grain sizes. Our method involves first computing the dispersion 
relation of Alfvén waves in the presence of a discrete set of grain sizes in the relevant low dust-to-gas mass ratio limit (see also Tripathi \& Sharma 1996; Cramer et al. 2002). A surprisingly simple expression for the continuum system is obtained by taking the limit as the total number of grain species approaches infinity, while keeping the total mass density of dust constant. Importantly, this expression scales with the total dust density, is effectively independent of the dust drag time, and converges rapidly to the continuum limit, implying that large damping and growth rates occur even with a continuum of grain sizes (unlike, e.g. the low dust-to-gas-ratio streaming instability; Krapp et al. 2019). A simple extension of the calculation shows that an (isothermal) dust pressure response does not change the damping or growth rate, suggesting that a dust velocity dispersion driven, e.g. by gas turbulence (Yan et al. 2004) - will not strongly affect the shear-Alfvénic modes of interest (although a true kinetic treatment is needed to formally probe this physics; see Appendix A3). Mathematical details of the calculation are given in Appendix A.

\subsection{Dust model and definitions}

We model dust as a charged, pressure-less fluid that interacts with the gas through drag and Lorentz forces. The gas is modelled with the ideal MHD equations, which is also a reasonable model for a collision-less plasma for the scales and modes being considered here (Schekochihin et al. 2009). Our equations do, however, assume that the total charge contained in the dust species is small compared to that of the background gas (Shukla \& Mamun 2002). For a set of $N_{d}$ dust species, labelled $i$, the equations are

$$
\begin{aligned}
& \frac{\partial \rho}{\partial t}+\nabla \cdot(\boldsymbol{u} \rho)=0, \\
& \frac{\partial \rho_{d, i}}{\partial t}+\nabla \cdot\left(\boldsymbol{v}_{i} \rho_{d, i}\right)=0, \\
& \frac{\partial \boldsymbol{u}}{\partial t}+\boldsymbol{u} \cdot \nabla \boldsymbol{u}=\boldsymbol{g}-\frac{1}{\rho} \nabla\left(p+\frac{B^{2}}{8 \pi}\right)-\frac{\boldsymbol{B} \cdot \nabla \boldsymbol{B}}{4 \pi \rho}, \\
& +\sum_{i=1}^{N_{d}} \frac{\rho_{d, i}}{\rho}\left(\frac{\boldsymbol{v}_{i}-\boldsymbol{u}}{t_{s, i}}-\frac{\boldsymbol{v}_{i}-\boldsymbol{u}}{t_{L, i}} \times \hat{\boldsymbol{b}}\right) \\
& \frac{\partial \boldsymbol{v}_{i}}{\partial t}+\boldsymbol{v}_{i} \cdot \nabla \boldsymbol{v}_{i}=\boldsymbol{g}+\mathbf{a}_{i}^{\mathrm{ext}}+\left(\frac{\boldsymbol{u}-\boldsymbol{v}_{i}}{t_{s, i}}-\frac{\boldsymbol{u}-\boldsymbol{v}_{i}}{t_{L, i}} \times \hat{\boldsymbol{b}}\right), \\
& \frac{\partial \boldsymbol{B}}{\partial t}+\boldsymbol{u} \cdot \nabla \boldsymbol{B}=\boldsymbol{B} \cdot \nabla \boldsymbol{u}-\boldsymbol{B} \nabla \cdot \boldsymbol{u} .
\end{aligned}
$$

Here $\rho, \boldsymbol{u}, p$, and $\boldsymbol{B}$ are respectively the gas density, velocity, pressure, and the magnetic field, while $\hat{\boldsymbol{b}}$ is the magnetic field unit vector $(\boldsymbol{B}=$ $B \hat{\boldsymbol{b}})$ and $\boldsymbol{g}$ is an external gravitational force on both gas and dust. We also define the Alfvén speed $v_{A}=B / \sqrt{4 \pi \rho}$. The continuum mass density and bulk velocity of dust species $i$ are $\rho_{d, i}$ and $\boldsymbol{v}_{i}$ respectively, and $\mathbf{a}_{i}^{\text {ext }}$ is an external force that can act differently on each dust species (e.g. due to radiation pressure). The physics of a particular dust species is determined by its microscopic parameters: the grain radius $a_{d, i}$, mass $m_{d, i}$, solid density $\bar{\rho}_{d, i}=3 m_{d, i} /\left(4 \pi a_{d, i}^{3}\right)$, charge $q_{d, i}=Z_{d, i} e$, and electrostatic potential $U_{d, i} \approx q_{d, i} / a_{d, i}$. These parameters determine the stopping time $t_{s, i}$, which is also a function of gas parameters (see Section 4.1.1), and the Larmor time

$t_{L, i}=\frac{m_{d, i} c}{q_{d, i} B}$,

which can be either positive or negative depending on the sign of $q_{d, i}$. We use $\langle\cdot\rangle$ to denote an equilibrium (background) quantity, and define the dust-to-gas mass ratio $\mu_{i}=\left\langle\rho_{d, i} / \rho\right\rangle$, and the total dust-to-gas-mass ratio $\mu_{0}=\sum_{i} \mu_{i}$.
As shown in HS18, in the presence of an external force on the dust $\left(\mathbf{a}_{i}^{\text {ext }} \neq 0\right)$, a formal quasi-equilibrium is set up in which both dust and gas accelerate at the same rate, but with some velocity offset, denoted $\mathbf{w}_{s}$. Moving into the frame in which the gas is stationary allows one to study instabilities about the equilibrium $\left\langle\boldsymbol{v}_{i}\right\rangle=\mathbf{w}_{s, i}$, $\langle\boldsymbol{u}\rangle=0$, with the free-energy source for the instabilities arising from the net drift of each species of dust. For arbitrary $t_{s, i} / t_{L, i}$, and when the angle between $\mathbf{a}_{i}^{\text {ext }}$ and the background magnetic field $\boldsymbol{B}_{0}$ is arbitrary, the expression for $\mathbf{w}_{s, i}$ is rather complex, arising from the balance between magnetic and drag forces on grains. However, as shown by HS18 (see their equation 2), in the limit $\left|t_{L, i}\right| \ll t_{s, i}, \mathbf{w}_{s, i}$ tends to align more and more closely with $\boldsymbol{B}_{0}$, albeit with a magnitude that is reduced by the projection factor $\left(\mathbf{a}_{i}^{\text {ext }} \cdot \boldsymbol{B}_{0}\right) /\left(\left|\mathbf{a}_{i}^{\text {ext }}\right|\left|\boldsymbol{B}_{0}\right|\right)$. Intuitively, this corresponds to the fact that well magnetized particles are only free to move along the magnetic field direction. Because most regimes of interest for the study of CR propagation satisfy $\left|t_{L, i}\right| \ll t_{s, i}$ (see HS18 fig. 6), we thus assume that $\mathbf{w}_{s, i}$ lies parallel to $\boldsymbol{B}_{0}$, simplifying the analysis enormously. The general case where $\mathbf{a}_{i}^{\text {ext }}$ and $\boldsymbol{B}_{0}$ are not parallel is thus effectively contained within our analysis by including some order-unity projection factor $\Phi \equiv\left(\mathbf{a}_{i}^{\text {ext }} \cdot \boldsymbol{B}_{0}\right) /\left(\left|\mathbf{a}_{i}^{\text {ext }}\right|\left|\boldsymbol{B}_{0}\right|\right)$, but without having to account for a complex, $3 \mathrm{D}$ equilibrium where dust drifts at an arbitrary angle to the magnetic field. So long as $\left|t_{L, i}\right| \ll$ $t_{s, i}$, this is a good approximation on time-scales longer than $\sim t_{s, i}$ (the time taken for the system to reach equilibrium).

\subsection{Damping and growth of parallel Alfvénic modes}

Our analysis proceeds in the standard way by linearizing equations (2) to (6) about the quasi-equilibrium described above: $\langle\boldsymbol{u}\rangle=0,\langle\rho\rangle$ $=\rho_{0},\left\langle\boldsymbol{v}_{i}\right\rangle=w_{s, i} \hat{z},\left\langle\rho_{d, i}\right\rangle=\mu_{i} \rho,\langle\boldsymbol{B}\rangle=\boldsymbol{B}_{0}=B_{0} \hat{z}$, where $w_{s, i}$ can be zero (stationary dust). We then insert the Fourier ansatz for the evolution of each linearized quantity $-\delta f(\boldsymbol{x}, t) \equiv f(\boldsymbol{x}, t)-\langle f\rangle=$ $\delta f \exp (i \boldsymbol{k} \cdot \boldsymbol{x}-i \omega t)$ for $f=\boldsymbol{u}, \rho$, etc. - to convert equations 2 to (6) into an eigenvalue equation for the frequency $\omega$. $\Im(\omega)<0(\Im(\omega)$ $>0$ ) implies that a particular mode is damped (growing). A further significant simplification comes from specializing to purely parallel modes $\boldsymbol{k}=k \hat{z}\left(k_{x}=k_{y}=0\right)$. The justification for this simplification is that CRs interact strongly only with purely parallel modes (Kulsrud \& Pearce 1969; Chandran 2000), so even if an oblique mode were to grow (or be damped) more rapidly, there will be little effect on CR scattering. In addition, parallel modes are usually the fastest growing in the $\left|t_{L}\right| \ll t_{s}$ regime (see HS18).

With these simplifications, it transpires that one can obtain accurate, simple forms for the dispersion relation $\omega(k)$ for modes with frequencies near the Alfvén frequency $\omega_{A}=k v_{A}$. Specifically, one inserts the ansatz $\omega=k v_{A}+\mu\left(\omega^{(1)} / \mu\right)$ (such that $\omega^{(1)}$ is the perturbed frequency), and expands the characteristic equation in the small parameter $\mu \ll 1$ with $\omega^{(1)} / \mu$ finite, taking $\mu_{i}=\bar{\mu}_{i} \mu$ (i.e. all individual dust densities $\mu_{i}$ scale with $\mu$ ). Solving the polynomial equation for $\omega^{(1)}$ that appears at lowest order in $\mu$ and taking its imaginary part leads to the result

$$
\begin{aligned}
\Im\left(\omega^{(1)}\right) & =\omega_{A} \sum_{i=1}^{N_{d}} \mu_{i} \tilde{\omega}_{i}^{(1)} \\
& =\omega_{A} \sum_{i=1}^{N_{d}} \mu_{i} \mathcal{W}_{s, i}^{2} \bar{t}_{s, i} \frac{\mathcal{W}_{s, i}^{2} \bar{t}_{s, i}^{2}\left(2 \bar{\lambda}_{i}^{2} \mathcal{W}_{s, i}+\bar{\lambda}_{i}^{2}-1\right)-1}{\left(\bar{\lambda}_{i}^{2}-1\right)^{2} \mathcal{W}_{s, i}^{4} \bar{t}_{s, i}^{4}+2\left(\bar{\lambda}_{i}^{2}+1\right) \mathcal{W}_{s, i}^{2} \bar{t}_{s, i}^{2}+1} .
\end{aligned}
$$

Here, $\mathcal{W}_{s, i} \equiv w_{s, i} / v_{A}-1$ is the relative Alfvén Mach number of the streaming dust and $\bar{t}_{s, i}=\omega_{A} t_{s, i}$ is the normalized stopping time. $\bar{\lambda}_{i}=k^{-1} / \lambda_{\text {res }, i}$ is the mode's inverse wavenumber normalized by the resonance wavelength $\lambda_{\text {res }, i} \equiv v_{A} t_{L, i} \mathcal{W}_{s, i}$, which is the inverse 
wavenumber of the Alfvén wave mode that matches the streaming gyro-orbit frequency of the dust; i.e. the wavelength for which $\omega_{A}=k w_{s, i}-t_{L, i}^{-1}$. As expected, the contribution of each grain species $\left(\tilde{\omega}_{i}^{(1)}\right.$ in equation 8$)$ is negative (damping) if $\mathcal{W}_{s, i}<0$ and positive (unstable) if $\mathcal{W}_{s}>0$.

To take the continuum limit of equation (8), we note that $\tilde{\omega}_{i}^{(1)}$ becomes increasingly sharply peaked around the resonance $\left(\bar{\lambda}_{i}=1\right)$ at increasing $\bar{t}_{s, i} \gg 1$. This implies that each species contributes to the total damping or growth rate only around the resonant wavenumber $\bar{\lambda}_{i}=1$. As shown in Appendix A, this allows for the straightforward derivation of a simple expression for continuum damping or growth rate,

$\Gamma_{\text {dust }}(k)=\left.\operatorname{sgn}\left(\mathcal{W}_{s}\right) \omega_{A} \mu_{0} \frac{\pi}{2} \frac{\mathrm{d} \bar{\mu}\left(a_{d}\right)}{\mathrm{d} \ln a_{d}} \frac{\mathcal{W}_{s}\left(a_{d}\right)^{2}}{\left|\xi_{t_{L}}+\xi_{\mathcal{W}_{s} \mid}\right|}\right|_{k^{-1}=v_{A} t_{L}\left(a_{d}\right) \mathcal{W}_{s}\left(a_{d}\right)}$.

Here, we have changed from labelling each dust species by its discrete index $i$, to making $\mathcal{W}_{s}$ and $t_{L}$ functions of physical grain size $a_{d}$, defining

$\xi_{t_{L}} \equiv \frac{\mathrm{d} \ln \left|t_{L}\right|}{\mathrm{d} \ln a_{d}}, \quad \xi_{\mathcal{W}_{s}} \equiv \frac{\mathrm{d} \ln \left|\mathcal{W}_{s}\right|}{\mathrm{d} \ln a_{d}}$.

The fractional mass across a given range of sizes is parametrized by $\mathrm{d} \bar{\mu} / \mathrm{d} \ln a_{d}$, which satisfies $\int \mathrm{d} \ln a_{d}\left(\mathrm{~d} \bar{\mu} / \mathrm{d} \ln a_{d}\right)=1$ across the full range of grain sizes present (i.e. $\mathrm{d} \bar{\mu} / \mathrm{d} \ln a_{d}$ is the fractional contribution to dust density from grains of size $\left.a_{d}\right){ }^{1}$ Evaluating equation (9) as a function of $k$ involves first inverting the resonance condition, $v_{A} t_{L}\left(a_{d}\right) \mathcal{W}_{s}\left(a_{d}\right)=k^{-1}$, to find $a_{d}(k)$, then inserting this into the main expression (9). More detail about the derivation of equations (8) and (9) and how they relate to the Resonant Drag Instability theory of Squire \& Hopkins (2018) and HS18 is given in Appendix A. We also confirm the validity of equation (9) in Fig. A1, by comparing to numerical solutions of the full dispersion relation for a discrete set of dust grains.

\subsubsection{Polarization}

Our derivation of equation (8) from the frequency alone has hidden the relevance of the mode polarization inside the sign of $t_{L}$, which controls the sign of $k$ (or $\bar{\lambda}_{i}$ ) and thus the mode propagation direction and polarization. Physically, only modes that resonate with the dust can interact with it, which, for negatively charged dust and waves propagating in the magnetic field direction, implies that only righthand polarized waves are damped for $\mathcal{W}_{s}<0$, while only lefthand polarized waves are unstable for $\mathcal{W}_{s}>0$. The opposite is true for positively charged dust and/or for wave propagation in the antimagnetic field direction. The handedness of the damped/unstable waves (determined by the dust charge) does not feature prominently in the discussion below, because the streaming speed of CRs $\left(\sim v_{A}\right)$ is generally much smaller than the speed of individual particles $(\sim c)$, implying there are nearly equal numbers of forward and backward propagating particles. However, the fact that only one polarization is damped or unstable does suggest some interesting implications on CR transport, as discussed in Section 2.1.

\section{ASTROPHYSICAL CONSEQUENCES}

In this section, we consider how the dust-induced damping or growth of parallel Alfvén waves (AWs) could impact CR confinement in

${ }^{1}$ For the standard MRN size distribution, $\mathrm{d} n_{d} \propto a^{-3.5} \mathrm{~d} a_{d}$ (Mathis, Rumpl \& Nordsieck 1977), $\mathrm{d} \bar{\mu} / \mathrm{d} \ln a_{d} \propto a_{d}^{0.5}$. galaxies. We suggest two possible effects, which have opposite consequences for CR propagation. The first - discussed in Section 4.2 - involves the additional AW damping caused by dust reducing the efficiency of CR self-confinement, thus enhancing the CR transport. The second - discussed in Section 4.3 - considers how dust with super-Alfvénic drift speeds in the circumgalactic medium (CGM) could excite parallel AWs that directly scatter CRs, thus enhancing the CR confinement (reducing the transport). We start with a brief review of the dust properties that will be necessary for our discussion, focusing on expressions and/or physical processes that are relevant to $a_{d} \lesssim 1 \mu \mathrm{m}$ grains in well-ionized gas with a temperature $T \gtrsim 10^{4} \mathrm{~K}$. This focus is motivated by the fact that in colder, predominantly neutral gas, CRs are thought to diffuse rapidly anyway due to strong ion-neutral damping, which will generally dominate the effects we discuss here for reasonable dust-to-gas mass ratios.

In addition to quantities defined above, throughout this section it will be convenient to use subscripts to denote orders of magnitude in cgs units with $F_{x}=F / 10^{x}$ for some quantity $F$; thus $T_{4}$ is the gas temperature in units of $10^{4} \mathrm{~K}, n_{0}$ is the gas number density in units of $\mathrm{cm}^{-3}, B_{-6}$ is the magnetic field strength in units of $\mu \mathrm{G}, a_{-5}$ is the grain radius $a_{d}$ in units of $0.1 \mu \mathrm{m}$, and $\bar{\rho}_{d ; 0}$ is the grain solid density in units of $\mathrm{g} \mathrm{cm}^{-3}$. We also define the plasma 'beta,' which is the ratio of thermal pressure to magnetic pressure $\beta=8 \pi P / B^{2}$.

\subsection{Physical properties of astrophysical dust}

The key dust properties of interest for computing the damping/growth rate of Alfvén waves from equation (9) are: (i) the dust drag law $t_{s}\left(a_{d}\right)$, which, in addition to the external force on the grains, determines $\mathcal{W}_{s}\left(a_{d}\right)$; (ii) the dust charge, which determines $t_{L}\left(a_{d}\right)$ from equation (7); and (iii) the mass distribution of grain sizes $\mathrm{d} \bar{\mu} / \mathrm{d} \ln a$. Let us discuss each of these in turn, summarizing relevant information from previous literature.

\subsubsection{Drag law}

The two relevant expressions for grains in the conditions of interest here are Epstein drag, which is collisional drag when the particle size is smaller than the gas mean free path, and Coulomb drag, which arises when charged grains interact with the background plasma (Draine \& Salpeter 1979b). The stopping time for grain species $i$ in the Epstein regime is approximately

$$
\begin{aligned}
t_{s, i}^{\mathrm{Ep}} & =\sqrt{\frac{\pi}{8}} \frac{\bar{\rho}_{d, i} a_{d, i}}{\rho v_{\mathrm{th}}}\left(1+\frac{9 \pi}{128} \frac{\left|\boldsymbol{v}_{i}-\boldsymbol{u}\right|^{2}}{v_{\mathrm{th}}^{2}}\right)^{-1 / 2} \\
& \approx 6.8 \times 10^{12} \mathrm{~s} \frac{a_{-5} \bar{\rho}_{d ; 0}}{n_{0} T_{4}^{1 / 2}},
\end{aligned}
$$

where $v_{\text {th }}$ is the gas thermal velocity, while in the Coulomb regime it follows

$$
\begin{aligned}
t_{s, i}^{\text {Coul }} & \approx \sqrt{\frac{\pi}{2}} \frac{\bar{\rho}_{d, i} a_{d, i}}{f_{\text {ion }} \rho v_{\text {th }} \ln \Lambda_{d}}\left(\frac{k_{B} T}{z_{i} e U_{d}}\right)^{2}\left(1+\sqrt{\frac{2}{9 \pi}} \frac{\left|\boldsymbol{v}_{i}-\boldsymbol{u}\right|^{3}}{v_{\text {th }}^{3}}\right), \\
& \approx 1.1 \times 10^{11} \mathrm{~s} \frac{a_{-5} \bar{\rho}_{d ; 0}}{f_{\text {ion }} n_{0} T_{4}^{1 / 2} \bar{U}^{2}},
\end{aligned}
$$

where $f_{\text {ion }}$ is the ionization fraction, $\ln \Lambda_{d}$ is the Coulomb logarithm for the dust ( $\ln \Lambda_{d} \approx 20$ for the conditions and grains of interest; see HS18; Draine \& Salpeter 1979b), $z_{i}$ is the mean gas ion charge, and $-2.5 \bar{U}=U_{d} /\left(k_{B} T / e\right) \approx e^{2} Z_{d} /\left(a_{d} k_{B} T\right)$ is the normalized grain potential (see Section 4.1.2 below). In the second lines of equations (11) and (12) we have assumed subsonic grain drift $\left|\boldsymbol{v}_{i}-\boldsymbol{u}\right| \ll v_{\text {th }}$, 
taken the mean molecular weight to be 0.6 (a typical choice for warm ionized gas), and taken $z_{i} \approx 1$. The correct drag law is approximately whichever of equations (11) and (12) is smaller. For maximally collisionally charged $(\bar{U} \approx 1)$ grains moving subsonically in well-ionized gas, this is Coulomb drag, otherwise Epstein drag dominates.

\subsubsection{Grain charge}

Grain charging processes are very complicated and uncertain, being strongly influenced by a range of environmental effects and grain microphysics. Further, in many systems there is expected to be a wide distribution of different grain charges for a given grain size. Here we use several different simple analytic fits to approximate the average charge expected in different regimes, as discussed in, e.g. Draine \& Salpeter (1979b), Draine \& Sutin (1987), Weingartner \& Draine (2001c), Weingartner (2004), Tielens (2005).

The simplest relevant process is collisional charging, which satisfies approximately

$U_{d} \approx \frac{e Z_{d}}{a_{d}} \approx-\frac{e}{a_{d}} \frac{1}{1+0.037 \sqrt{\frac{e^{2}}{a k_{B} T}}}-2.5 \frac{k_{B} T}{e} \approx-2.5 \frac{k_{B} T}{e}$,

where the latter approximation is valid for the conditions of interest $\left(T \gtrsim 10^{4} \mathrm{~K}\right)$. This motivates defining $-2.5 \bar{U}=U_{d} /\left(k_{B} T / e\right)$, with $\bar{U} \approx 1$ for collisionally charged grains. For reference, the grain potential is $U_{d} \approx-2.15 T_{4} \bar{U} \mathrm{~V}$. When $Z_{d}$ becomes too negative, the total charge becomes limited by electron field emission to $Z_{d} \approx-7000 a_{-5}^{2}$, which translates to

$\bar{U} \approx \bar{U}_{0} \min \left\{1,47 \frac{a_{-5}}{T_{4}}\right\}$,

where we retain a dimensionless $\bar{U}_{0} \approx 1$ factor, so as to understand the charge dependence of analytic expressions that we later derive. At higher gas temperatures $\left(T \gtrsim 10^{5} \mathrm{~K}\right.$ for silicate grains, $T \gtrsim 10^{5.5} \mathrm{~K}$ for graphite), secondary electron emission can cause grains to become positively charged. This process can be important, especially for smaller grains where it can lead to larger $\left|U_{d}\right|$ than the standard collisional expression (14); but, we do not consider it in detail because of the complex, material-dependent expressions (see figs 1 and 2 of Draine \& Salpeter 1979b).

In the presence of a radiation field, photoelectric charging can dominate, which also causes grains to gain net positive charge. The process depends primarily on the gas/radiation through the parameter, $\psi=G_{0} T^{1 / 2} / n$, where $G_{0}=u_{\mathrm{rad}}^{\mathrm{uv}} /\left(5.3 \times 10^{-14} \mathrm{erg} \mathrm{cm}^{-3}\right)$ and $u_{\mathrm{rad}}^{\mathrm{uv}}$ is the energy density in the radiation field between $\sim 6$ and $13.6 \mathrm{eV}$. The charge is approximately $Z_{d} \approx 36(\psi / 1000) a_{-5}$ up to a maximum potential $U \sim 7 \mathrm{~V}$ or $Z_{d} \approx 500 a_{-5}$ (see Weingartner \& Draine 2001c; Tielens 2005; Draine 2010 and fig. 4 of Weingartner 2004). This gives

$\bar{U} \approx-\bar{U}_{0} T_{4}^{-1} \min \left\{0.24\left(\frac{\psi}{1000}\right), 3.3\right\}$.

Overall, we see that in most regimes, $\bar{U}$ may be assumed independent of $a_{d}$ in order to compute the $\mathcal{W}_{s}$ and $t_{L}$ derivatives required to evaluate damping/growth of Alfvén waves (equation 9), while for small grains in hot gas without a strong radiation field, $\bar{U} \propto a_{d}$. For simplicity, we will neglect the quantization of grain charge in analytic estimates, although this does become significant for the smallest grains (see e.g. fig. 25.3 of Draine 2010).

\subsubsection{Mass distribution}

Grain mass distributions probably vary significantly between regions, depending on the complex interplay between grain growth, grain shattering through collisions, and grain sputtering from the gas (Peters et al. 2017). The standard MRN distribution of Mathis et al. (1977) postulates that $\mathrm{d} \bar{\mu} / \mathrm{d} \ln a_{d} \propto a_{d}^{0.5}$ with $a_{d, \min } \approx 5 \mathrm{~nm}\left(a_{-5}=\right.$ $0.05)$ and $a_{d, \max } \approx 0.25 \mu \mathrm{m}\left(a_{-5}=2.5\right)$, and a total dust-to-gas mass ratio of around 1 per cent $\left(\mu_{0}=0.01\right)$. The MRN distribution likely has significant inaccuracies even in the ISM, missing a sizeable population of small grains with $a_{d}<5 \mathrm{~nm}$ (e.g. Weingartner \& Draine 2001a; Zubko, Dwek \& Arendt 2004; Draine \& Fraisse 2009). Far less is known about the CGM or hotter regions. Grain destruction due to ion-field emission is expected to be significant only for very small grains in very hot gas, and not strongly affect our results here (e.g. in $T \sim 10^{6} \mathrm{~K}$ gas; Draine \& Salpeter $1979 \mathrm{~b}$ suggest the ion-fieldemission-limited grain size is $a \lesssim 1 \mathrm{~nm}$ ). Thermal sputtering rates become larger than grain growth due to accretion of metals from the gas for $T \gtrsim 10^{5} \mathrm{~K}$, and significant compared to gas dynamical times for $T \gtrsim 10^{6} \mathrm{~K}$ (Draine \& Salpeter 1979a,b), suggesting that the dust-to-gas mass ratio may be lower in hotter gas. However, dust is clearly observed in the CGM of galaxies (Ménard et al. 2010; Peek, Ménard \& Corrales 2015) and thus appears to survive in gas up to $T \sim 10^{7} \mathrm{~K}$, although this may also be related to the presence of multiphase gas (i.e. dust existing primarily in clumps of colder gas; Tumlinson, Peeples \& Werk 2017). While its size distribution remains very uncertain, the presence of smaller grains (down to at least $a_{d} \sim 0.01 \mu \mathrm{m}$ ) is indicated by observations (Hirashita \& Lin 2020).

Given these very significant uncertainties, the use of a complex dust-size distribution model would be of dubious value, ${ }^{2}$ and for simplicity we will use a power-law grain mass distribution $\mathrm{d} \bar{\mu} / \mathrm{d} \ln a_{d} \propto a_{d}^{\xi_{\mu}}$ between $a_{d, \min } \approx 1 \mathrm{~nm}$ and $a_{d, \text { max }} \approx 0.25 \mu \mathrm{m}$ in all estimates, with $\xi_{\mu}=0.5$ (MRN) as the fiducial choice. Since wave growth/damping rates around a particular wavelength scale linearly with the density of dust that is resonant with that wavelength, one can simply adjust the total dust-to-gas mass ratio $\mu_{0}$ to account for uncertainty in the mass distribution.

\subsection{Enhanced cosmic ray transport in the warm ionized ISM}

Here we ask the question of whether dust damping, as derived in Section 3, can compete with turbulent processes and non-linear Landau damping to damp parallel waves and enable the fast transport of CRs (see Section 2). This simply involves directly computing the damping rate from equation (9) to compare with these previously studied processes. We compare our expressions graphically, for a range of reasonable warm-ISM parameters, in Fig. 1.

\subsubsection{Dust-induced damping}

In the bulk of the ISM, radiation-pressure forces are relatively weak and grain drift is expected to be significantly sub-Alfvénic (Weingartner \& Draine 2001b; see fig. 6 of HS18). It is thus reasonable to set $w_{s}=0$, or $\mathcal{W}_{s}=-1$ in evaluating equation (9), which also implies that $t_{s}$ does not directly enter the analysis

${ }^{2}$ Further, the mass distribution of grains is important for wave damping/growth only in so far as it determines the mass density of grains with a given $t_{L}, \mathcal{W}_{s}$, and $t_{s}$. This implies that the mass distribution gets mixed with the charge distribution, which has its own significant uncertainties. 

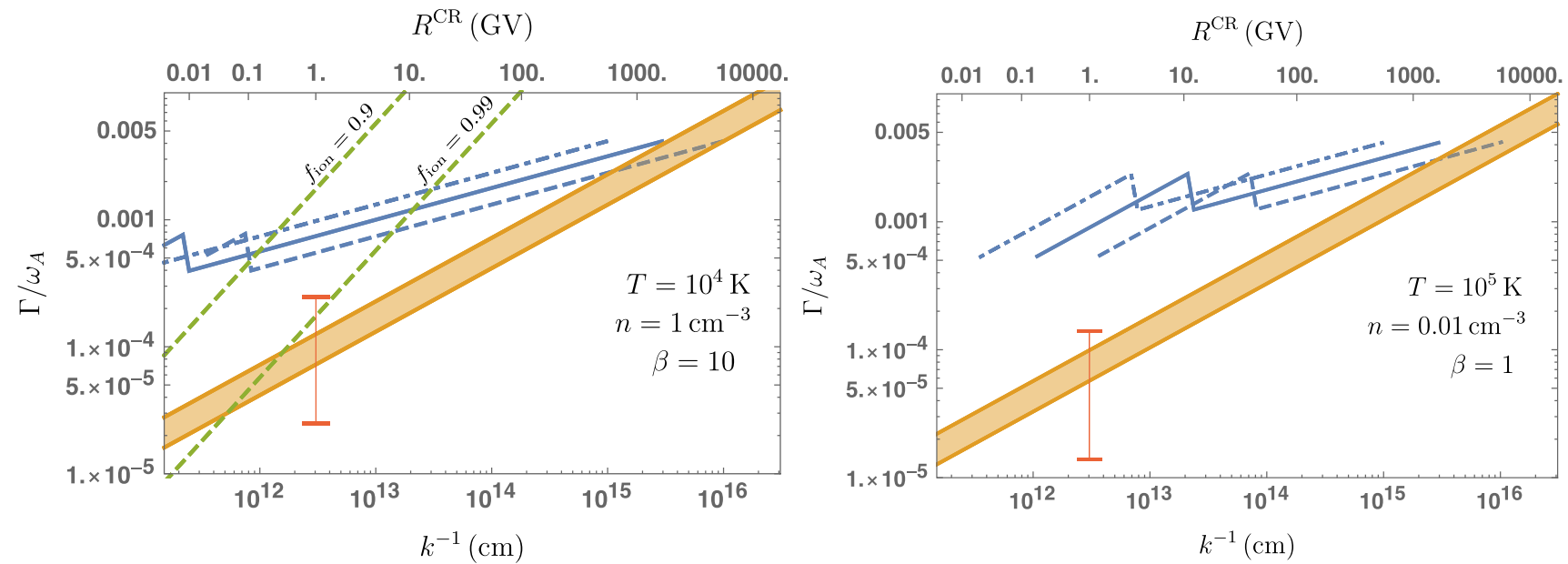

Figure 1. Comparison of parallel AW damping due to dust (blue lines; equation 18), turbulent damping (yellow region; equation 25), non-linear Laudau damping (red bar; equation 27), and ion-neutral damping (green line; equation 28) as a function of scale. The top axis shows the rigidity of CRs that are resonant with AWs at $k^{-1}$ for the conditions of interest. The left-hand panel illustrates cooler, denser conditions, with $T=10^{4} \mathrm{~K}, n=1 \mathrm{~cm}^{-3}$, and $\beta=10$, while the right-hand panel illustrates hotter, more diffuse conditions $T=10^{5} \mathrm{~K}, n=0.01 \mathrm{~cm}^{-3}$, and $\beta=1$. For the dust, the total dust-to-gas mass ratio is $\mu_{0}=0.01$, and grains range between $a_{d, \min }=0.01 \mu \mathrm{m}, a_{d, \min }=0.25 \mu \mathrm{m}$ with a mass distribution $\mathrm{d} \bar{\mu} / \mathrm{d} \ln a \propto a^{0.5}$. We consider collisional charging (equation 14), and the different lines show the effect of changing grain charge with $\bar{U}_{0}=1$ (solid line), $\bar{U}_{0}=0.3$ (dashed line), $\bar{U}_{0}=3$ (dot-dashed line). Lines terminate at the small and large scales given by equation (20). For the turbulent damping, the shaded region shows the range for $l_{\text {turb }}$ in the range 100 to $300 \mathrm{pc}$ with $M_{A} \approx$ 1. For non-linear Landau damping, we indicate the damping rate for the energetically dominant $\sim \mathrm{GeV} \mathrm{CRs}$, which, because it is non-linear, depends on the $\mathrm{CR}$ properties and transport model. For basic comparison, we use $l_{\mathrm{CR}}=300 \mathrm{pc}$ and $e_{\mathrm{CR}}$ and in the range 0.1 to $10 \mathrm{eV} \mathrm{cm}^{-3}\left(e_{\mathrm{CR}} \approx 0.3 \mathrm{eV} \mathrm{cm}{ }^{-3}\right.$ is measured around the solar circle). Although our focus is on CR transport in well-ionized gas, we also show ion-neutral damping rates with $f_{\text {ion }}=0.9$ and $f_{\text {ion }}=0.99$ in the left-hand panel (green dashed lines) in order to allow for a basic comparison.

(although we must ensure $t_{S} \omega_{A} \gg 1$ for the modes of interest for equation (9) to be valid). For concreteness, we start by considering grain charging in the absence of a radiation field (equation 14), which gives the Larmor time (equation 7)

$t_{L} \approx-1.7 \times 10^{9} \mathrm{~s} \frac{a_{-5}^{2} \bar{\rho}_{d ; 0}}{\bar{U} T_{4} B_{-6}}$.

Dust will damp CR-induced AWs so long as the scales that are resonant with CRs are also resonant with grains present in the ISM. The former resonant-CR scale, $k_{\mathrm{res}}^{-1} \approx r_{L}$, is given by equation (1), while the dust-resonant wavenumber $k^{-1}=v_{A}\left|t_{L}\right|$ is

$k^{-1}\left(a_{d}\right)=3.3 \times 10^{12} \mathrm{~cm}\left\{\begin{array}{ll}\left(\frac{a_{d}}{8.2 \mathrm{~nm}}\right)^{2} \frac{\bar{\rho}_{d ; 0}}{n_{0}^{1 / 2} T_{4} \bar{U}_{0}} & k \lesssim k_{\mathrm{efe}} \\ \left(\frac{a_{d}}{31 \mathrm{~nm}}\right) \frac{\bar{\rho}_{d ; 0}}{n_{0}^{1 / 2} \bar{U}_{0}} & k \gtrsim k_{\mathrm{efe}}\end{array}\right.$,

where we have normalized the grain size to illustrate the connection to scales relevant for $R^{\mathrm{CR}} \approx 1 \mathrm{GV}$ protons (see equation 1 ). Here $k_{\mathrm{efe}}^{-1} \approx$ $\left(2.2 \times 10^{11} \mathrm{~cm}\right) T_{4} \bar{\rho}_{d ; 0} /\left(n_{0}^{1 / 2} \bar{U}_{0}\right)$ is the resonant scale at which the grain charging changes from the collisional regime (large scales; $a_{-5}$ $\gtrsim T_{4} / 47$ ) to the electron-field-emission-limited regime (small scales; $a_{-5} \lesssim T_{4} / 47$ ) (see equation 14 ). We also see from equation (10) that $\xi_{t_{L}} \approx 2$ for $a_{-5} \gtrsim T_{4} / 47$ (larger scales), and $\xi_{t_{L}} \approx 1$ for $a_{-5} \lesssim T_{4} / 47$ (smaller scales), showing that the damping rate is somewhat larger in the electron-field-emission-limited regime. ${ }^{3}$ It is straightforward to verify that $t_{S} \omega_{A} \gg 1$ for all grains (Coulomb drag dominates; equation 12).

The growth rate is computed by inverting equation (17) for $a_{d}$ and using the normalized power-law grain mass distribution $\mathrm{d} \bar{\mu} / \mathrm{d} \ln a_{d}=$

${ }^{3}$ This occurs because the Larmor time increases more slowly with grain size, meaning there are more grains that are resonant with a particular wavelength of wave. $\xi_{\mu} a_{d}^{\xi_{\mu}} /\left(a_{d, \max }^{\xi_{\mu}}-a_{d, \min }^{\xi_{\mu}}\right)$. equation (9) then gives

$$
\begin{aligned}
& \frac{\Gamma_{\text {dust }}}{\omega_{A}} \approx \frac{\pi}{4} \frac{\mu_{0}}{\Delta_{a_{d}}^{\xi_{\mu}}} \begin{cases}\left(\frac{k^{-1}}{4.9 \times 10^{14} \mathrm{~cm}}\right)^{\xi_{\mu} / 2}\left(\frac{n_{0}^{1 / 2} T_{4} \bar{U}_{0}}{\bar{\rho}_{d ; 0}}\right)^{\xi_{\mu} / 2} & k_{a_{d, \text { max }}} \lesssim k \lesssim k_{\text {efe }} \\
2\left(\frac{k^{-1}}{1.0 \times 10^{13} \mathrm{~cm}}\right)^{\xi_{\mu}}\left(\frac{n_{0}^{1 / 2} \bar{U}_{0}}{\bar{\rho}_{d ; 0}}\right)^{\xi_{\mu}} & k_{a_{d, \text { min }}} \gtrsim k \gtrsim k_{\text {efe }}\end{cases} \\
& \approx \begin{cases}7.6 \times 10^{-4}\left(\frac{\mu_{0}}{0.01}\right)\left(\frac{k^{-1}}{3.3 \times 10^{12} \mathrm{~cm}}\right)^{1 / 4} \frac{n_{0}^{1 / 8} T_{4}^{1 / 4} \bar{U}_{0}^{1 / 4}}{\bar{\rho}_{d ; 0}^{1 / 4}} & k_{a_{d, \text { max }}} \lesssim k \lesssim k_{\mathrm{efe}} \\
3.0 \times 10^{-3}\left(\frac{\mu_{0}}{0.01}\right)\left(\frac{k^{-1}}{3.3 \times 10^{12} \mathrm{~cm}}\right)^{1 / 2} \frac{n_{0}^{1 / 4} \bar{U}_{0}^{1 / 2}}{\bar{\rho}_{d ; 0}^{1 / 2}} & k_{a_{d, \min }} \gtrsim k \gtrsim k_{\mathrm{efe}}\end{cases}
\end{aligned}
$$

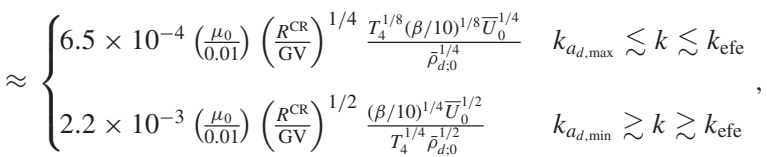

where $\Delta_{a_{d}}^{\xi_{\mu}}=\left[\left(a_{d, \max } / 0.1 \mu \mathrm{m}\right)^{\xi_{\mu}}-\left(a_{d, \min } / 0.1 \mu \mathrm{m}\right)^{\xi_{\mu}}\right] / \xi_{\mu} \approx 3.0$ for grains in the range $a_{d, \min } \approx 1 \mathrm{~nm}$ to $a_{d, \max } \approx 0.25 \mu \mathrm{m}$ with $\xi_{\mu}$ $=0.5$, which is used to simplify the expressions from the second line. equation (1) is used to convert between scale and CR rigidity for the third line (assuming relativistic CRs, $R^{\mathrm{CR}} \gtrsim 1 \mathrm{GV}$ ). The wavenumber cutoffs, $k_{a_{d, \max }}$ and $k_{a_{d, \text { min}}}$, are the wavenumbers resonant with the largest and smallest grains, respectively. Renormalizing $a_{d}$ in equation (17), we find

$k_{a_{d, \min }}^{-1} \approx 1.0 \times 10^{11} \mathrm{~cm} \frac{\bar{\rho}_{d ; 0}}{n_{0}^{1 / 2} \bar{U}_{0}}\left(\frac{a_{d, \min }}{1 \mathrm{~nm}}\right)$,

$k_{a_{d, \max }}^{-1} \approx 3.1 \times 10^{15} \mathrm{~cm} \frac{\bar{\rho}_{d ; 0}}{T_{4} n_{0}^{1 / 2} \bar{U}_{0}}\left(\frac{a_{d, \max }}{0.25 \mu \mathrm{m}}\right)^{2}$,

assuming that $a_{d}=a_{d \text {, min }}$ grains are in the field-emission-limited regime, and $a_{d}=a_{d, \max }$ grains are in the collisional regime. We see that the resonant scales of grains, where there is strong damping, 
covers a similar range to that of $\sim \mathrm{GeV}$ CRs across a wide range of parameters relevant to the well-ionized ISM. Note that equation (18) is discontinuous across $k \approx k_{\text {efe }}$ (larger at small scales), because $\xi_{t_{L}}$ changes.

In many regions of the ISM, while radiation pressures may not be sufficiently large to drive grains to super-Alfvénic velocities, the radiation field can none the less be strong enough for photoelectric charging to dominate over collisional charging (e.g. Weingartner \& Draine 2001b; Draine 2010). In this case grains become positively charged (equation 15) to several volts depending on the strength of the radiation field. Aside from the polarization of the damped waves, which makes no difference to the CR transport within our approximations (see Section 2.1), the calculation of the wavedamping rate is nearly identical except that there is no electronfield-emission limited regime. Taking $\bar{U}=-\bar{U}_{0} / T_{4}$ (corresponding to $U_{d} \approx 2.2 \mathrm{~V} \bar{U}_{0}$ ), with $\bar{U}_{0}$ encapsulating our ignorance about the radiation field, the same calculation as above gives the dust-resonant wavenumber

$k^{-1}\left(a_{d}\right)=3.3 \times 10^{12} \mathrm{~cm}\left(\frac{a_{d}}{8.2 \mathrm{~nm}}\right)^{2} \frac{\bar{\rho}_{d ; 0}}{n_{0}^{1 / 2} \bar{U}_{0}}$,

with damping rate

$$
\begin{aligned}
\frac{\Gamma_{\text {dust }}}{\omega_{A}} & \approx 7.6 \times 10^{-4}\left(\frac{\mu_{0}}{0.01}\right)\left(\frac{k^{-1}}{3.3 \times 10^{12} \mathrm{~cm}}\right)^{1 / 4} \frac{n_{0}^{1 / 8} \bar{U}_{0}^{1 / 4}}{\bar{\rho}_{d ; 0}^{1 / 4}} \\
& \approx 6.5 \times 10^{-4}\left(\frac{\mu_{0}}{0.01}\right)\left(\frac{R^{\mathrm{CR}}}{\mathrm{GV}}\right)^{1 / 4} \frac{(\beta / 10)^{1 / 8} \bar{U}_{0}^{1 / 4}}{T_{4}^{1 / 8} \bar{\rho}_{d ; 0}^{1 / 4}}
\end{aligned}
$$

between the minimum and maximum scales,

$k_{a_{d, \text { min }}}^{-1} \approx 4.9 \times 10^{10} \mathrm{~cm} \frac{\bar{\rho}_{d ; 0}}{n_{0}^{1 / 2} \bar{U}_{0}}\left(\frac{a_{d, \min }}{1 \mathrm{~nm}}\right)^{2}$,

$k_{a_{d, \max }}^{-1} \approx 3.1 \times 10^{15} \mathrm{~cm} \frac{\bar{\rho}_{d ; 0}}{n_{0}^{1 / 2} \bar{U}_{0}}\left(\frac{a_{d, \max }}{0.25 \mu \mathrm{m}}\right)^{2}$,

which is nearly identical to the collisionally charged result above. Of course, it is plausible that in some regions photo-electric and collisional charging will cancel out, in which case $|\bar{U}|$ could be reduced substantially compared to the estimates made above; however, it is reasonable to expect that in most regions one of the two charging regimes should dominate over the other.

\subsubsection{Other damping mechanisms}

Equation (18) should be compared to the damping rate of parallel AWs from other processes. The most important is turbulent damping, which arises from the fact that parallel AWs excited by CRs must propagate along inhomogenous turbulent magnetic fields. Using the Goldreich \& Sridhar (1995) phenomenology of anisotropic magnetized turbulence, the damping rate of $r_{L} \approx k^{-1}$ scale waves is (Farmer \& Goldreich 2004; Lazarian 2016; Zweibel 2017)

$$
\begin{aligned}
\frac{\Gamma_{\text {turb }}}{\omega_{A}} & \approx \frac{1}{\omega_{A}} \frac{v_{A}}{r_{L}^{1 / 2} l_{A}^{1 / 2}} \\
& \approx 1.0 \times 10^{-4} \Lambda_{\beta}\left(\frac{k^{-1}}{3.3 \times 10^{12} \mathrm{~cm}}\right)^{1 / 2} M_{A}^{1 / 2 \zeta}\left(\frac{l_{\text {turb }}}{100 \mathrm{pc}}\right)^{-1 / 2} \\
& \approx 7.6 \times 10^{-5} \Lambda_{\beta}\left(\frac{R^{\mathrm{CR}}}{\mathrm{GV}}\right)^{1 / 2}\left(\frac{\beta / 10}{n_{0} T_{4}}\right)^{1 / 4} M_{A}^{1 / 2 \zeta}\left(\frac{l_{\text {turb }}}{100 \mathrm{pc}}\right)^{-1 / 2}
\end{aligned}
$$

Here $l_{\text {turb }}$ is the outer scale of the turbulence and $l_{A}=M_{A}^{-1 / \zeta} l_{\text {turb }}$ is the scale at which turbulent fluctuations become sub-Alfvénic, with $M_{A}$ the Alfvén Mach number at $l_{\text {turb }}$ and $\delta u \sim l^{-\zeta}$ the powerlaw turbulent scaling exponent of $l>l_{A}$ motions $(\zeta \sim 1 / 2$ for supersonic motions, or $\zeta \sim 1 / 3$ for subsonic motions). The factor $\Lambda_{\beta} \approx \max (1,0.4 \sqrt{\beta})$ arises because at lower $\beta$, direct turbulent dissipation of perpendicular waves is expected to dominate (Farmer $\&$ Goldreich 2004), while at larger $\beta$, linear-Landau damping of perpendicular magnetosonic waves dominates (Zweibel 2017; Wiener, Zweibel \& Oh 2018). Although Alfvénic turbulence is known to be quite robust across a wide range of plasma conditions, it is worth noting that any other processes that enhance the damping of turbulence beyond these standard estimates (e.g. Silsbee, Ivlev \& Gong 2020) would reduce small-scale fluctuations in the magnetic field, thus decreasing $\Gamma_{\text {turb }}$ and the importance of turbulence to $\mathrm{CR}$ propagation.

Non-linear Landau damping (NLLD) is the process by which nonlinear magnetic field strength variations in the small-scale parallel AWs are directly damped by resonant particle interactions (Lee \& Völk 1973; Cesarsky \& Kulsrud 1981; Völk \& Cesarsky 1982) and pressure anisotropy (Squire, Quataert \& Schekochihin 2016; Squire, Schekochihin \& Quataert 2017). As a non-linear effect, the strength of the damping depends on the amplitude of the waves as

$\Gamma_{\mathrm{NLL}} \approx \frac{\sqrt{\pi}}{8} v_{\mathrm{th}} k \frac{\delta \boldsymbol{B}^{2}(k)}{B^{2}}$,

where the wave amplitude $\delta \boldsymbol{B}^{2}(k) / B^{2}$ itself depends sensitively on the CR energy density and its gradient. In order to make a basic comparison to other mechanisms, we use convenient expressions from $\mathrm{H}+20$ (equation A4) for the damping rate of waves resonant with $\sim \mathrm{GeV}$ CRs. These are derived using an approximate balance of growth and damping, assuming that the waves are excited by CRs with energy density $e_{\mathrm{CR}}$ that varies over length scale $l^{\mathrm{CR}}$ (see also Thomas \& Pfrommer 2019), giving

$$
\begin{aligned}
\frac{\Gamma_{\mathrm{NLL}}}{\omega_{A}} \approx & \frac{1}{\omega_{A}}\left[\frac{1}{3} \frac{\pi^{1 / 2}}{8}\left(\frac{c_{s} v_{A}}{r_{L} l_{\mathrm{CR}}}\right)\left(\frac{e_{\mathrm{CR}}}{B^{2} / 8 \pi}\right)\right]^{1 / 2} \\
\approx & 1.4 \times 10^{-4}\left(\frac{k^{-1}}{3.3 \times 10^{12} \mathrm{~cm}}\right)^{1 / 2} \frac{(\beta / 10)^{3 / 4}}{n_{0}^{1 / 2} T_{4}^{1 / 2}} \\
& \times\left(\frac{e_{\mathrm{CR}}}{1 \mathrm{eV} \mathrm{cm}^{-3}}\right)^{1 / 2}\left(\frac{l_{\mathrm{CR}}}{100 \mathrm{pc}}\right)^{-1 / 2} .
\end{aligned}
$$

However, estimating the rigidity dependence of this damping rate requires a rigidity-dependent model of $\mathrm{CR}$ transport (which manifests in equation 27 through the rigidity dependence of $e_{\mathrm{CR}}$ ). While we suggest a way to estimate this below (Section 4.2.3), leading to the scaling $\Gamma_{\mathrm{NLL}} / \omega_{A} \propto\left(R^{\mathrm{CR}}\right)^{0.15}$, given the greater uncertainty in these estimates, in Fig. 1 we plot only the damping rate for $\sim \mathrm{GeV}$ CRs. In any case, equation (27) and the results of $\mathrm{H}+20$ suggest that NLLD is subdominant and unimportant in most situations, being overwhelmed by turbulent damping even in the absence of dust.

Finally, although we do not consider partially ionized gas and ion-neutral damping in detail, it is helpful to include for comparison purposes. For waves with frequencies below the ion-neutral collision rate $v_{\text {in }}$, the AW damping rate is $(\mathrm{H}+20$; Amato \& Blasi 2018)

$$
\frac{\Gamma_{\mathrm{in}}}{\omega_{A}} \approx \frac{\nu_{\mathrm{in}}}{\omega_{A}} \approx 0.04\left(\frac{k^{-1}}{3.3 \times 10^{12} \mathrm{~cm}}\right)\left(1-f_{\mathrm{ion}}\right) f_{\mathrm{ion}}^{1 / 2} \frac{T_{4}^{1 / 2} \rho_{-24}^{3 / 2}}{B_{-6}},
$$

where $\rho_{-24}=\rho /\left(10^{-24} \mathrm{~g} \mathrm{~cm}^{-3}\right)$ (mass density of both ions and neutrals), and the $f_{\text {ion }}^{1 / 2}$ factor arises because $v_{A}$ is a function of the ion density alone at small scales in a partially ionized gas 
(note that we have neglected this effect in equations (25) to (27) because it is unimportant if $\left.1-f_{\text {ion }} \ll 1\right)$. It is clear that ion-neutral damping dominates over all others when the neutral fraction $1-f_{\text {ion }}$ is modestly large.

\subsubsection{Implications for cosmic ray transport}

A graphical comparison of equations (18) to (28) in two sets of conditions relevant to the warm ISM, across a wide range of scales, is shown in Fig. 1. A clear conclusion is that the dust damping rate of AWs with a $\sim 1$ per cent dust-to-gas mass ratio is significantly larger than any other source of damping for AWs resonant with $\sim \mathrm{GeV}$ CRs (top axes), except ion-neutral damping with $1-f_{\text {ion }} \gtrsim 0.03$. In other words, dust may significantly decrease CR confinement in well-ionized regions of the ISM. The same conclusion is illustrated across a wider range of gas conditions in Fig. 2, which shows the ratio of dust to turbulent damping at the GeV CR gyro-scale, with solid contours illustrating the resonant grain size (assuming collisional charging). We see that it is generally very small grains $\left(a_{d} \lesssim 10 \mathrm{~nm}\right.$ for common ionized-ISM conditions) that are resonant with scales relevant for $\sim \mathrm{GeV}$ CRs.

Assuming that dust damping does dominate as suggested by the estimates above, we can balance the dust damping rate (18) to the streaming instability growth rate to obtain an estimate for the effective speed of CR energy transport, $\bar{v}_{\text {st }}$, in the presence of dust (see e.g. $\mathrm{H}+20$ section 3.3 ). For collisionally charged grains (equation 18), one finds

$$
\begin{aligned}
\bar{v}_{\mathrm{st}} & \approx v_{A}+\frac{3}{4} \frac{\kappa_{\|}}{l_{\mathrm{CR}}} \approx v_{A}+\frac{4 c r_{L}}{\pi} \frac{\Gamma_{\mathrm{damp}}}{v_{A}} \frac{B^{2}}{8 \pi} \frac{1}{e_{\mathrm{CR}}} \\
& \approx v_{A}\left\{\begin{array}{ll}
1+4.1\left(\frac{R^{\mathrm{CR}}}{1 \mathrm{GV}}\right)^{1 / 4}\left(\frac{e_{\mathrm{CR}}}{1 \mathrm{eV} \mathrm{cm}^{-3}}\right)^{-1}\left(\frac{\mu_{0}}{0.01}\right) \frac{n_{0} T_{4}^{5 / 8} \bar{U}_{0}^{1 / 4}}{(\beta / 10)^{3 / 8} \bar{\rho}_{d ; 0}^{1 / 4}} & R^{\mathrm{CR}} \gtrsim R_{\mathrm{efe}}^{\mathrm{CR}} \\
1+14\left(\frac{R^{\mathrm{CR}}}{1 \mathrm{GV}}\right)^{1 / 2}\left(\frac{e_{\mathrm{CR}}}{1 \mathrm{le} \mathrm{cm}^{-3}}\right)^{-1}\left(\frac{\mu_{0}}{0.01}\right) \frac{n_{0} T_{4}^{1 / 4} \bar{U}_{0}^{1 / 2}}{(\beta / 10)^{1 / 4} \bar{\rho}_{d ; 0}^{1 / 2}} & R^{\mathrm{CR}} \lesssim R_{\mathrm{efe}}^{\mathrm{CR}}
\end{array},\right.
\end{aligned}
$$

where $\kappa_{\|}=c^{2} / 3 v_{c}$ is the parallel CR diffusion coefficient and $R_{\text {efe }}^{\mathrm{CR}} \approx 0.4 T_{4}^{3 / 2} \bar{\rho}_{d ; 0} /\left(\bar{U}_{0} \beta^{1 / 2}\right) \mathrm{GV}$ is the CR rigidity corresponding to $k_{\text {efe }}$. Similarly, for photoelectrically charged grains (equation 22) one finds

$$
\begin{aligned}
\bar{v}_{\mathrm{st}} \approx & v_{A}\left[1+4.1\left(\frac{R^{\mathrm{CR}}}{1 \mathrm{GV}}\right)^{1 / 4}\left(\frac{e_{\mathrm{CR}}}{1 \mathrm{eV} \mathrm{cm}{ }^{-3}}\right)^{-1}\right. \\
& \left.\times\left(\frac{\mu_{0}}{0.01}\right) \frac{n_{0} T_{4}^{3 / 8} \bar{U}_{0}^{1 / 4}}{(\beta / 10)^{3 / 8} \bar{\rho}_{d ; 0}^{1 / 4}} \cdot\right]
\end{aligned}
$$

Note that equations (29) and (30) apply only to the $\sim \mathrm{GeV}$ CRs that dominate the $\mathrm{CR}$ energy density, because of the appearance of $e_{\mathrm{CR}}$ in the expressions, and we also assume relativistic CRs to convert scales to rigidity using equation (1). Evidently, we predict modestly super-Alfvénic CR energy transport with faster transport at higher temperature, lower $\beta$, or with more strongly charged grains. It is, however, worth noting that this streaming speed likely remains too small to fully explain the discrepancy found by $\mathrm{H}+20$ between observations and cosmological simulations with self-confined CRs (or equivalently, the AW damping rate remains too small). Further simulations with a dust-damping self-confinement model would be needed to test this more thoroughly.

Finally, we can estimate the predicted rigidity dependence of self-confined CR transport regulated by dust, $\tilde{v}_{\mathrm{st}} \propto\left(R^{\mathrm{CR}}\right)^{\delta}$ (or equivalently $\left.\kappa_{\|} \propto\left(R^{\mathrm{CR}}\right)^{\delta}\right)$. Such predictions can be compared to the measured rigidity dependence of $\mathrm{CR}$ grammage from, e.g. the

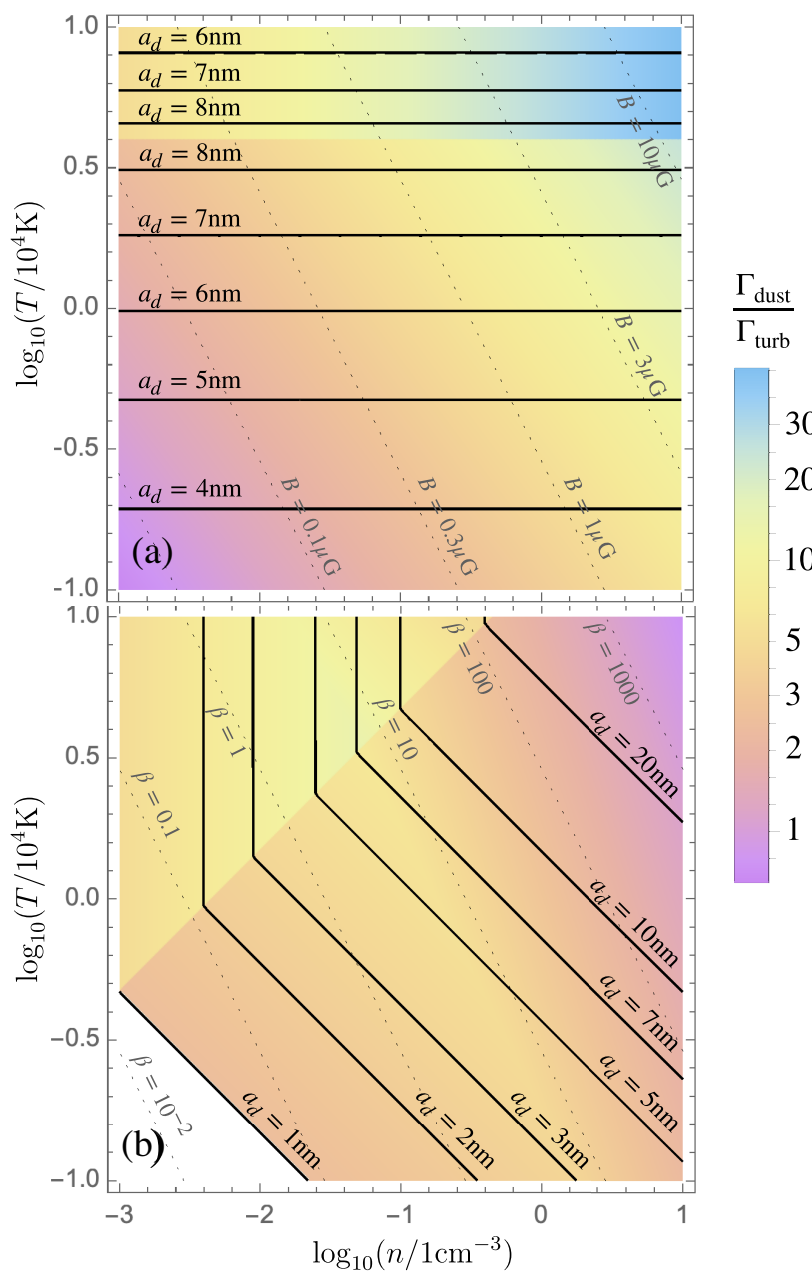

Figure 2. Colours illustrate the ratio $\Gamma_{\text {dust }} / \Gamma_{\text {turb }}$ of the dust damping rate to turbulent damping rate for scales corresponding to $R^{\mathrm{CR}}=1 \mathrm{GV} \mathrm{CRs}$, across a wide range of temperatures and densities relevant to the ionized ISM. Collisional charging (equation 18) is assumed for $\Gamma_{\text {dust }}$. The top panel keeps $\beta=10$ constant as $n$ and $T$ are changed, while the bottom panel keeps $B=1 \mu \mathrm{G}$ constant. In each panel, the solid contours show the size of the resonant grains (equation 17) as labelled, while the light, dashed contours show lines of constant $B$ (top panel) or constant $\beta$ (bottom panel). The step change in colour in each panel $\left(T_{4} \approx 10^{0.6}\right.$ in the top panel; the diagonal from $T_{4} \approx 10^{-0.3}$ in the bottom panel) is where the charging becomes electron field emission limited, and the white region in the bottom panel occurs because the resonant scale moves below $a=1 \mathrm{~nm}$. Other relevant parameters are the same as Fig. 1 , with $\bar{U}_{0}=1$ and $l_{\text {turb }}=100 \mathrm{pc}$.

$\mathrm{B} / \mathrm{C}$ ratio, which suggests $\delta$ in the range 0.3 to 0.8 (with various uncertainties related to sources and propagation; Maurin, Putze \& Derome 2010; Amato 2014; Blasi 2017; Aguilar et al. 2018). To estimate $\delta$, we follow the kinetic treatment of Skilling (1971), which, by balancing the damping rate of AWs to the growth rate of the streaming instability, arrives at the the following equation governing the distribution $f(p)$ of CRs of momentum $p$ :

$$
\left(\frac{\partial}{\partial t}+\boldsymbol{w} \cdot \nabla\right) f=\frac{1}{3} p \frac{\partial f}{\partial p} \nabla \cdot \boldsymbol{w}-\frac{1}{p^{3}} \nabla \cdot\left(\frac{c \Gamma_{\mathrm{damp}}(p) B}{4 \pi^{3} e v_{A}} \hat{\boldsymbol{b}}\right),
$$

where $\boldsymbol{w}=\boldsymbol{u}+v_{A} \hat{\boldsymbol{b}}$ is the velocity of the AW frame, in which equation (31) is valid. The wave-damping rate $\Gamma_{\text {damp }}$ has an effective 
momentum dependence ${ }^{4}$ because it is in general a function of $k$, and $k$ and $p$ are related by the CR resonance condition equation (1). We see that the final 'damping' term on the right-hand side, which necessarily describes any deviation from transport at exactly $\tilde{v}_{\mathrm{st}}=v_{A}$ within these approximations, does not have the form of an advection or diffusion operator at all, and in fact does not even contain the CR distribution $f$ (its rather bizarre behaviour is discussed in Skilling 1971). None the less, as a very simple, approximate estimate for the scaling of an effective transport speed $\tilde{v}_{\text {st }}$ with CR momentum, we can compare this damping term to an advection term, which would have the form $\tilde{v}_{\mathrm{st}} \hat{\boldsymbol{b}} \cdot \nabla f$. Noting that $f \sim p^{-\alpha}$ with $\alpha \approx 4.7$, while $\Gamma_{\text {damp }}$ scales as $\Gamma_{\text {damp }} \sim k^{b} \sim p^{-b}$, we can estimate $\delta$ by matching the $p$ scaling of $\tilde{v}_{\mathrm{st}} \hat{\boldsymbol{b}} \cdot \nabla f$ to that of $p^{-3} \Gamma_{\text {damp }}$, giving $\delta \approx \alpha-3$ $-b=1.7-b$. From equation (18), we see $b=1-\xi_{\mu} / 2 \approx 0.75$, giving $\delta \approx 0.7+\xi_{\mu} / 2 \approx 0.95$. Although this estimate is a little higher than observationally favoured values, it is worth noting that the other AW damping mechanisms except non-linear-Landau damping ${ }^{5}$ all predict larger $\delta$ (e.g. $\delta \approx 1.2$ for turbulence damping). A flatter (or even inverted) mass distribution of small grains, as favoured by more detailed models of dust (see Section 4.1.3), would decrease $\delta$ further.

\subsubsection{Uncertainties}

The most significant uncertainties in the above conclusions relate to the fact that a reasonable population of small grains $\left(a_{d} \lesssim 10 \mathrm{~nm}\right)$ is required to damp waves relevant to $\sim \mathrm{GeV} \mathrm{CRs}$. If the total dust-togas mass ratio were significantly lower than $0.1 \rightarrow 0.3$ per cent in more diffuse ISM regions (see e.g. Peters et al. 2017), or if the grain size distribution were significantly depleted for $a_{d} \lesssim 10 \mathrm{~nm}$, dust damping could be unimportant compared to other sources. However, it is worth noticing that most complex dust models that are calibrated to observations (e.g. Weingartner \& Draine 2001a; Zubko et al. 2004; Draine \& Fraisse 2009) involve a grain distribution with an excess of the smallest grains compared to a power-law distribution, which suggests that using $\xi_{\mu}=0.5$ could underestimate the dust damping, as well as overestimating the dependence of transport on CR rigidity $(\delta)$. Further uncertainty concerns the charging of small grains, which will be affected by charge quantization (Draine 2010) as well as the environmental factors discussed above (e.g. the radiation environment); a more accurate treatment could convolve a charge distribution with the mass distribution before computing the damping rate to account for quantization. Finally, we reiterate that only one wave polarization is damped by dust (see Section 3.2.1), so that in practice, dust tends to make CR-induced AWs more circularly polarized. As discussed in Section 2.1, this likely enhances CR transport by a similar degree to what would occur if both polarizations were damped; however, simulations and more accurate theory are clearly needed to assess this physics in more detail.

\footnotetext{
${ }^{4}$ Skilling (1971) neglects the $k$ dependence of $\Gamma_{\text {damp }}$ in his calculation. Including it yields slightly different (unimportant) numerical factors in the final term of equation (31).

${ }^{5}$ The scaling of NLLD can be worked through in the same way, with the caveat that $\Gamma_{\text {damp }}$ depends directly on the CR spectrum in Skilling's argument. This leads to a damping term that scales as $\sim p^{-3 / 2} \nabla \cdot\left[A(\hat{\boldsymbol{b}} \cdot \nabla f)^{1 / 2} \hat{\boldsymbol{b}}\right]$ (where $A$ represents terms that depend on gas quantities), which gives $\delta=0.85$. This also implies that $\Gamma_{\mathrm{NLL}}$ from equation (27) scales as $\Gamma_{\mathrm{NLL}} \sim k^{0.85}$ for this model of CR transport. For comparison, this is slightly flatter than the other mechanisms plotted in Fig. 1. This calculation is effectively the same as that of Blasi (2019), who also finds $\delta=0.85$ for NLLD-mediated transport.
}

\subsection{Enhanced cosmic ray scattering through dust-excited Alfvén waves}

In this section, we discuss the possibility that dust drifting with $\left|\mathbf{w}_{s}\right|>$ $v_{A}$ generates small-scale parallel Alfvén waves, which subsequently scatter and confine cosmic rays. Much of this physics remains highly uncertain; both the astrophysics of dust in the relevant conditions (e.g. charging and grain abundances in hotter gas) and the non-linear wave physics (e.g. saturation of the dust instability and CR scattering; see Section 2.1) are not well constrained. We thus provide only a cursory examination of the relevant physics compared to Section 4.2 on dust damping. None the less, in some situations - particularly when grains are driven to significantly super-Alfvénic velocities by radiation pressure - it is reasonable to suggest that dust-induced scattering rates could dominate over all other CR scattering mechanisms, causing scattering levels near the 'Bohm limit,' where the CR's meanfree path approaches their gyro-radius. Here, motivated by HS18, we consider the interesting example of the circumgalactic medium (CGM) around a quasar or highly luminous galaxy, which could cause an interesting non-linear correlation between the luminosity and the confinement of CRs. Other possible applications include near supernovae ${ }^{6}$ and regions of the ISM with higher-than-average radiation fields (see e.g. fig. 6 and section 9 of HS18), although the dust instabilities may become more complicated in some cases where grains are not so strongly magnetized (if the ratio $\left|t_{L}\right| / t_{s}$ approaches one, see Section 3.1).

As shown in Section 3, positively charged dust drifting at speeds exceeding $v_{A}$ will generate parallel Alfvén waves. If their growth rate exceeds the damping rate from background turbulence, ${ }^{7}$ such waves will presumably continue to grow until they saturate through some other means. The most obvious candidate is by exciting some type of turbulence, which scatters dust particles sufficiently to shut off the instability's drive. The simulations of Hopkins et al. (2020c) suggest that a simple saturation criterion is when a characteristic turnover time at the scale of interest is equal to the instability growth rate, which leads to an estimate of the power in small scale parallel fluctuations $\delta \boldsymbol{B}^{2} / B^{2} \sim\left(\Gamma / \omega_{A}\right)^{2}$, where $\Gamma / \omega_{A}$ is the growth rate of the dust instability normalized by the Alfvén frequency at the chosen scale. This implies the CR diffusivity

$\kappa_{\|} \approx \frac{c^{2}}{3 v_{c}} \sim 3.3 \times 10^{26} \mathrm{~cm}^{2} \mathrm{~s}^{-1}\left(\frac{R^{\mathrm{CR}}}{\mathrm{GV}}\right)\left(\frac{B}{1 \mu \mathrm{G}}\right)^{-1}\left(\frac{\Gamma / \omega_{A}}{0.01}\right)^{-2}$.

It is worth noting that this estimate $\delta \boldsymbol{B}^{2} / B^{2} \sim\left(\Gamma / \omega_{A}\right)^{2}$ likely significantly underestimates the saturation level of small-scale AWs, because the instability grows with circularly polarized modes that do not perturb the field strength, so will not necessarily breakup into turbulent fluctuations in the usual way. On the other hand, it is also likely the case that pure circularly polarized modes are very inefficient at isotropizing CRs (see Section 2.1), so equation (32) could be a reasonable estimate of effective scatterers (i.e. turbulent fluctuations of both polarizations) even if one polarization reaches higher amplitude. Unfortunately, the extremely small scales of interest were not directly probed by the simulations of Hopkins et al. (2020c) (the 'CGM' simulation approaches similar

\footnotetext{
${ }^{6}$ Of course, CR fluxes are also particularly extreme near supernovae, and the relevant processes remain highly uncertain (Bykov et al. 2018; Micelotta, Matsuura \& Sarangi 2018; Holcomb \& Spitkovsky 2019).

${ }^{7}$ Non-linear Landau damping will presumably not operate on the dust-excited AWs, since they do not involve a variation in $|\boldsymbol{B}|$.
} 
conditions). Further simulations or more detailed analytic theory are clearly needed, but we proceed anyway for lack of a more accurate estimate.

We now focus on conditions relevant to the CGM around a quasar for concreteness, although similar considerations could apply to any high-luminosity galaxy. We first note that significant amounts of dust is observed in the CGM (Ménard et al. 2010; Peek et al. 2015), presumably driven there by radiation pressure (e.g. Ishibashi \& Fabian 2015; Hirashita \& Inoue 2019). Considering conditions appropriate to distances $r \sim r_{100} 100 \mathrm{kpc}$ from a quasar of luminosity $L \sim L_{13} 10^{13} L_{\odot}$, we take the gas to be hot $\left(T \sim 10^{5} \rightarrow 10^{7} \mathrm{~K}\right)$, at very low density $\left(n \sim 10^{-6} \rightarrow 10^{-3} \mathrm{~cm}^{-3}\right)$, and with weak magnetic fields $\left(\beta \sim 100 \rightarrow 10^{4}\right) .{ }^{8}$ The first stage of estimating the AW growth-rate from equation (9) is to estimate the Larmor time and relative drift velocity of grains. Estimates of $\psi$ indicate that grains will be strongly photoelectrically charged (equation 14 ), suggesting $\bar{U} \approx 3 / T_{4}\left(U_{d}\right.$ $\sim 7 \mathrm{~V}$ ) and that Epstein drag dominates (particularly given that we find trans-sonic to supersonic drift velocities; c.f. equations 11 and 12). We estimate the radiative force on the grains as $m_{d} \mathrm{a}^{\text {ext }} \sim Q_{\mathrm{abs}} \pi a_{d}^{2} L /\left(4 \pi r^{2} c\right)$, where $Q_{\mathrm{abs}}$ is the absorption efficiency of the grains, which, for a radiative flux peaked around wavelength $\lambda_{\text {rad }}$, is $Q_{\text {abs }} \sim a_{d} / \lambda_{\text {rad }}$ for $a_{d} \lesssim \lambda_{\text {rad }}$ or $Q_{\text {abs }} \sim 1$ for larger grains (Weingartner \& Draine 2001c). Assuming, for simplicity, that the relative dust-gas drift is supersonic $\left(w_{s} \gg c_{s}\right)$ to solve $w_{s}=\mathrm{a}^{\text {ext }} t_{s}^{\mathrm{Ep}}$ for $w_{s}$, we find

$$
\frac{w_{s}}{v_{A}} \approx \mathcal{W}_{s} \approx 330 a_{-5}^{1 / 2} \frac{L_{13}^{1 / 2}}{r_{100}} B_{-8}{ }^{-1} \Phi
$$

for dust with $a_{d} \lesssim \lambda_{\text {rad }}$ and a spectrum that peaks around Lymanalpha wavelengths, $\lambda_{\text {rad }}=122 \mathrm{~nm}$ (we define $B_{-8}=B / 10^{-8} \mathrm{G}$ ). The projection factor $\Phi \equiv\left(\mathbf{a}^{\text {ext }} \cdot \boldsymbol{B}_{0}\right) /\left(\left|\mathbf{a}^{\text {ext }}\right|\left|\boldsymbol{B}_{0}\right|\right)$ accounts for a possible misalignment of the magnetic field with the radiative flux that accelerates grains (see Section 3.1). equation (34) somewhat overestimates $w_{s}$ for the smallest grains that are trans- or sub-sonic $\left(w_{s} / v_{A} \lesssim \beta^{1 / 2}\right)$, as well as for the largest grains with $a_{d} \gtrsim \lambda_{\text {rad }}\left(Q_{\text {abs }}\right.$ saturates at $Q_{\text {abs }} \sim 1$ ); these effects are correctly accounted for in Fig. 3. Equation (34) also does not allow for possible non-linear modifications to $w_{s}$ as the grains are scattered by the turbulence they induce (although this is likely a modest effect in this very short wavelength range; Moseley, Squire \& Hopkins 2019). The Larmor time is $t_{L} \approx\left(5.3 \times 10^{10} \mathrm{~s}\right) a_{-5}^{2} \bar{\rho}_{d ; 0} /\left(\bar{U}_{0} B_{-8}\right)$ using the photoelectric expression (15) for the grain charge. Inverting the resonant condition $k^{-1}=v_{A} t_{L} \mathcal{W}_{s}$ to link the grain size to wavelength, and assuming $\xi_{\mu}=0.5$ for $a_{d, \min } \approx 1 \mathrm{~nm}$ to $a_{d, \max } \approx 0.25 \mu \mathrm{m}$, equation (9) becomes

$$
\begin{aligned}
\frac{\Gamma}{\omega_{A}} \approx & 0.13\left(\frac{\mu_{0}}{100}\right)\left(\frac{k^{-1}}{3.3 \times 10^{14} \mathrm{~cm}}\right)^{3 / 5} \frac{(\beta / 300)^{7 / 10}}{n_{-4}^{2 / 5} T_{6}^{7 / 10}} \\
& \times\left(\frac{L_{13}}{r_{100}^{2}}\right)^{7 / 10}\left(\frac{\bar{U}_{0}}{\bar{\rho}_{d ; 0}}\right)^{3 / 5} \Phi^{7 / 5}
\end{aligned}
$$

\footnotetext{
${ }^{8} \mathrm{~A}$ potential complication and uncertainty is that the observed dust may not be cospatial with the hot gas in the CGM, which is known to be multiphase and clumpy (Tumlinson et al. 2017). In such a case, although our estimates could still apply to waves in the cooler, denser gas (suggesting high CR scattering rates therein), isolated clumpy regions with high scattering rates are likely to be ineffective at confining CRs (since the CRs fill the space between clumps; $\mathrm{H}+20$ ). On the other hand, most dust grains drift supersonically through the gas (equation 33), so will presumably blow through different gas phases, even if initially present only in a cooler phase. Clearly, the relevance of multiphase gas represents yet another significant uncertainty for this mechanism.
}

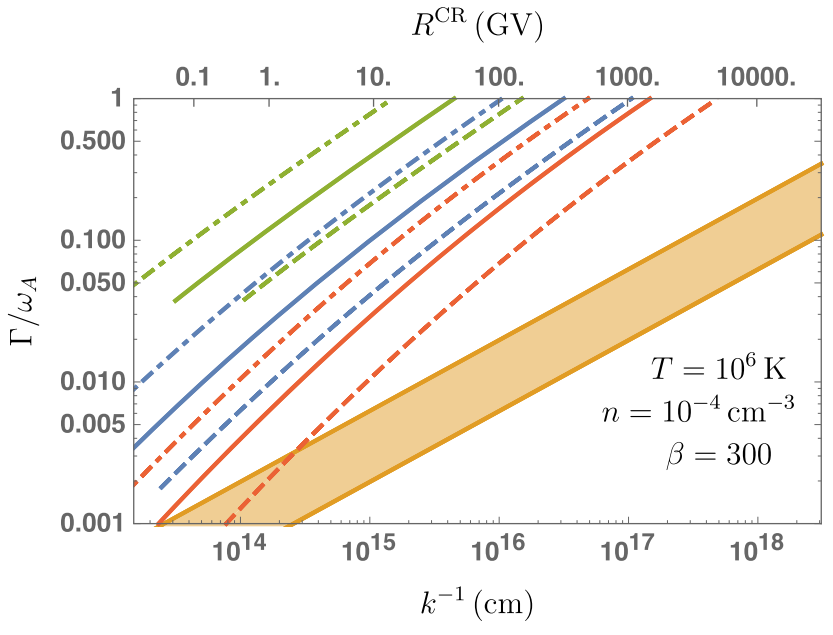

Figure 3. The growth rate of unstable AWs in the presence of drifting dust (lines) compared to turbulent damping rates (orange shaded region), for conditions relevant to the CGM around a luminous quasar $\left(T=10^{6} \mathrm{~K}, n\right.$ $\left.=10^{-4} \mathrm{~cm}^{-3}, \beta=300\right)$. We take $\mu_{0}=0.01$ with photoelectrically charged grains and an MRN grain spectrum between $a_{d, \min }=1 \mathrm{~nm}$ and $a_{d, \max }=$ $0.25 \mu \mathrm{m}$. The blue, green, and red lines, respectively, show growth rates with an $L \approx 10^{13} L_{\odot}, L \approx 5 \times 10^{13} L_{\odot}$, and $L \approx 3 \times 10^{12} L_{\odot}$ source, all at $r$ $\sim 100 \mathrm{kpc}$. As in Fig. 1, solid, dot-dashed, and dashed lines respectively show $\bar{U}=1, \bar{U}=3$, and $\bar{U}=0.3$, to illustrate the effect of changing the grain charge, and the lines terminate on the left at the scale where $a_{d \text {, min }}=$ $1 \mathrm{~nm}$ grains are resonant (equation 35). We take $\Phi=1$ since its scaling is degenerate with $L$ or $r$. For turbulent damping, we use the expression (25), with the range indicating the a range of outer-scales from $l_{\text {turb }} \sim 10 \mathrm{kpc}$ to $l_{\text {turb }} \sim 100 \mathrm{kpc}$ with $M_{A} \sim 5$.

where we have normalized $k^{-1}$ to the scale resonant with $\sim \mathrm{GeV}$ particles at $B=10^{-8} \mathrm{G}$. equation (34) applies on scales $k<k_{a_{d, \min }}$ where resonant grains exist, with

$k_{a_{d, \min }}^{-1} \approx 1.5 \times 10^{13} \mathrm{~cm}\left(\frac{a_{d, \min }}{1 \mathrm{~nm}}\right)^{5 / 2} \frac{(\beta / 300)^{1 / 2}}{n_{-4} T_{6}^{1 / 2}} \frac{L_{13}^{1 / 2}}{r_{100}} \frac{\bar{\rho}_{d ; 0}}{\bar{U}_{0}} \Phi$.

We see that growth rates are rather large at the relevant scales for $\sim \mathrm{GeV} \mathrm{CRs}$, and, at modestly larger scales relevant to $\gtrsim 50 \mathrm{GeV}$ particles, the scattering level might be expected reach Bohm diffusion levels (this occurs when $\delta \boldsymbol{B} / \boldsymbol{B} \sim 1$ from $\Gamma \sim \omega_{A}$, although our solution for $\Gamma$ breaks down here also). However, as also occurred for dust damping in the ISM (Section 4.2), it is the smaller grains that grow AWs at $\sim \mathrm{GeV}$ scales, and if the grain spectrum were cut off at small scales, or grains were less charged than expected, the spectrum of unstable AWs may not extend to sufficiently small scales to efficiently scatter $\sim \mathrm{GeV}$ particles.

We illustrate the AW growth rate for the fiducial conditions $(T=$ $10^{6} \mathrm{~K}, n=10^{-4} \mathrm{~cm}^{-3}, \beta=300$ ) with $\mu_{0}=0.01$ in Fig. 3 (although $\mu_{0}$ $=0.01$ may be an overestimate, the growth rate simply scales linearly with $\left.\mu_{0}\right)$. We use the full Epstein-drag expression to compute $w_{s}$, without assuming $w_{s} \gtrsim c_{s}$ or $a_{d} \lesssim \lambda_{\text {rad }}$ as in equation (33), which implies that the curves are slightly below the estimate (34) across most scales. Overall, we see significant dust-induced AW growth rates that are large compared to the effect of turbulence (shaded region), which provides a basic measure of the expected growth rate of the CR streaming instability in such regions. A caveat is that in order to scatter $\sim \mathrm{GeV}$ particles, there must be a population of quite small grains. Comparing the estimate of CR diffusivity in equation (32) with the estimate of $\kappa_{\|} \gtrsim 10^{30} \mathrm{~cm}^{2} \mathrm{~s}^{-1}$ in quiescent milky-waylike galaxies (based on $\gamma$-ray observations and simulations; see Lacki 
et al. 2011; Chan et al. 2019; $\mathrm{H}+20$ ), we see that reasonable quasar luminosities will cause very effective $\mathrm{CR}$ confinement, with a strong $\left(\sim L^{1.4}\right)$ dependence on luminosity up to the Bohm limit (at which point $\left.\kappa_{\|} \sim 3 \times 10^{24} \mathrm{~cm}^{2} \mathrm{~s}^{-1} B_{-8}^{-1}\left(R^{\mathrm{CR}} / 1 \mathrm{GV}\right)\right)$.

Such a strong increase in confinement may in turn have consequences for CR-driven winds or outflows. A crude measure of the impact is to compare the force per unit mass on the gas due to the dust (i.e. radiation pressure) to that due to the CRs. The former is $\rho_{d} w_{s} / t_{s} \approx 6.7 \times 10^{-38} \mathrm{gcm}^{-2} \mathrm{~s}^{-2}\left(\mu_{0} / 0.01\right) L_{13} n_{-4} /\left(r_{100}^{2} \bar{\rho}_{d ; 0}\right)$ assuming a supersonic dust-gas drift as above $\left(w_{s} / t_{s}\right.$ is approximately independent of $a_{d}$ for $\left.a_{d} \lesssim \lambda_{\text {rad }}\right)$; the latter is $\nabla P_{\mathrm{CR}}=e_{\mathrm{CR}} /\left(3 l_{\mathrm{CR}}\right) \approx$ $1.7 \times 10^{-38} \mathrm{gcm}^{-2} \mathrm{~s}^{-2}\left(e_{\mathrm{CR}} / 0.01 \mathrm{eV} \mathrm{cm}^{-3}\right)\left(l_{\mathrm{CR}} / 100 \mathrm{kpc}\right)^{-1}$. We see that even for relatively conservative values for $e_{\mathrm{CR}}$ and $l_{\mathrm{CR}}-$ i.e. values similar to those obtained with $\kappa_{\|} \gtrsim 10^{30} \mathrm{~cm}^{2} \mathrm{~s}^{-1}$ in $\mathrm{H}+20$ - the $\mathrm{CR}$ force is not vastly smaller than the radiation-pressure force. Thus, even a modest increase in CR confinement caused by dust-induced scattering would likely cause the force from CRs to overwhelm that due to radiation pressure, since $e_{\mathrm{CR}}$ or $1 / l_{\mathrm{CR}}$ need only increase by a factor of several for this to happen. In other words, this indirect effect of radiation pressure as a feedback mechanism for galaxies - driving small-scale waves that enhance CR confinement to the point where they drive outflows - may be more efficient than the direct driving of outflows through radiation pressure and dust drag (Murray, Quataert \& Thompson 2005). Under appropriate conditions, this could provide a strong feedback mechanism controlled by the luminosity of the quasar or galaxy.

\section{CONCLUSION}

This paper has considered the influence of astrophysical dust grains on the confinement of galactic cosmic rays (CRs). The cause of the interaction between these two seemingly unrelated components of the galactic ecosystem is the small-scale parallel Alfvén wave. Parallel Alfvén waves at sub-AU scales act to scatter and confine $\sim \mathrm{GeV}$ CRs through gyro-resonant interactions and the streaming instability. They also interact strongly - also through gyro-resonant interactions - with small charged dust grains, which act to damp the waves if the dust is nearly stationary with respect to the gas, or cause instability if the dust streams super-Alfvénically. This interaction implies a link between grain size (which determines the dust gyrofrequency through the dust mass and charge) and the CR rigidity: smaller grains, which are gyro-resonant with smaller wavelength AWs, interact with lower rigidity CRs.

The two possibilities (wave damping or instability from the dust) could have opposite effects on the astrophysics of cosmic rays. In the case of nearly stationary dust, for which the magnitude of the wave damping can be estimated with reasonable fidelity in well-ionized regions of the ISM, dust could significantly enhance CR transport (decrease confinement). Using reasonable assumptions about dust mass distributions and charging, we find damping rates from dust up to an order of magnitude larger than wave damping from the background turbulence (Farmer \& Goldreich 2004; Zweibel 2017) or non-linear damping (Cesarsky \& Kulsrud 1981). Because wave damping directly determines transport levels for self-confined CRs, this suggests that CR diffusion coefficients could be significantly larger than what would be expected without dust. The influence of dust may thus go some way towards explaining recent findings that standard models of CR self-confinement are too efficient to explain gamma-ray and in-situ CR grammage observations $(\mathrm{H}+20$; Chan et al. 2019; Hopkins et al. 2020d).

In the opposite case of dust-driven instability, the scattering of CRs by waves produced by dust has the potential to significantly reduce
CR transport (enhance confinement), although our estimates are far less reliable in this case due to significant astrophysical uncertainties. The situation could occur in the circumgalactic medium around a luminous galaxy, where radiation pressure would cause dust to stream outwards with super-Alfvénic relative velocities, exciting small-scale Alfvén waves that scatter CRs. Simple estimates suggest that the outwards force on the gas produced by the enhanced coupling to CRs through this interaction could be much stronger than the direct force from the dust/radiation pressure itself (Murray et al. 2005).

In either of the above scenarios, the relative mass density of dust plays a key role in determining $\mathrm{CR}$ transport, which thus implies a dependence of CR confinement on metallicity. In the first case of wave damping through dust, as most relevant to the ionized ISM away from high-luminousity sources, we predict more efficient CR escape in high-metallicity environments, because AWs are more rapidly damped at higher dust densities. The second scenario, more relevant to haloes around highly luminous galaxies, predicts the opposite, with stronger confinement at high metallicity. In this context, it is worth mentioning the particularly weak CR confinement in the SMC and LMC, as inferred from $\gamma$-ray observations (Lacki et al. 2011). Since the SMC and LMC have a low metallicity compared to the Milky Way, this does not fit with the first scenario of increased dust-induced damping, although given the wide array of other morphological and historical differences, the lower CR confinement is likely unrelated to the detailed CR transport physics (Chan et al. 2019). A metallicity dependence to CR transport could be a key ingredient in understanding the efficacy of CR feedback at high redshift.

\section{ACKNOWLEDGEMENTS}

Support for JS was provided by Rutherford Discovery Fellowship RDF-U001804 and Marsden Fund grant UOO1727, which are managed through the Royal Society Te Apārangi. Support for PFH was provided by NSF Collaborative Research Grants 1715847 \& 1911233,NSF CAREER grant 1455342, and NASA grants 80NSSC18K0562 and JPL 1589742. EQ was supported in part by NSF grant AST-1715070 and a Simons Investigator award from the Simons Foundation. We wish to acknowledge the hospitality of the Kavli Institute for Theoretical Physics during the program 'Multiscale Phenomena in Plasma Astrophysics,' which was supported in part by the National Science Foundation under Grant No. NSF PHY-1748958.

\section{DATA AVAILABILITY STATEMENT}

No new data were generated or analysed in support of this research.

\section{REFERENCES}

Adriani O. et al., 2011, Science, 332, 69

Aguilar M. et al., 2018, Phys. Rev. Lett., 120, 021101

Ahn H. S. et al., 2010, ApJ, 714, L89

Amato E., 2014, Inter. J. Mod Phys. D, 23, 1430013

Amato E., Blasi P., 2018, Adv. Space Res., 62, 2731

Bai X.-N., Ostriker E. C., Plotnikov I., Stone J. M., 2019, ApJ, 876, 60

Bell A. R., 2013, Astroparticle Phys., 43, 56

Blasi P., 2017, MNRAS, 471, 1662

Blasi P., 2019, Galaxies, 7, 64

Bustard C., Zweibel E. G., 2020, preprint (arXiv:2012.06585)

Butsky I. S., Quinn T. R., 2018, ApJ, 868, 108

Bykov A. M., Ellison D. C., Marcowith A., Osipov S. M., 2018, Space Sci. Rev., 214, 41 
Byleveld S. E., Melrose D. B., Cram L. E., 1993, Publ. Astron. Soc. Austr., 10,218

Cesarsky C. J., Kulsrud R. M., 1981, Origin Cosmic Rays, 94, 251

Chan T. K. et al., 2019, MNRAS, 488, 3716

Chandran B. D. G., 2000, Phys. Rev. Lett., 85, 4656

Cramer N. F., Verheest F., Vladimirov S. V., 2002, Phys. Plasmas, 9, 4845

Draine B. T., Fraisse A. A., 2009, ApJ, 696, 1

Draine B. T., Salpeter E. E., 1979a, ApJ, 231, 438

Draine B. T., Salpeter E. E., 1979b, ApJ, 231, 77

Draine B. T., Sutin B., 1987, ApJ, 320, 803

Draine B., 2010, Physics of the Interstellar and Intergalactic Medium, Princeton Series in Astrophysics. Princeton Univ. Press, Princeton

Dubois Y., Commerçon B., Marcowith A. r., Brahimi L., 2019, A\&A, 631, A121

Evoli C. et al., 2017, J. Cosmol. Astropart. Phys., 2017, 015

Farber R., Ruszkowski M., Yang H. Y. K., Zweibel E. G., 2018, ApJ, 856, 112

Farmer A. J., Goldreich P., 2004, ApJ, 604, 671

Felice G. M., Kulsrud R. M., 2001, ApJ, 553, 198

Goldreich P., Sridhar S., 1995, ApJ, 438, 763

Griffin R. D., Dai X., Thompson T. A., 2016, ApJ, 823, L17

Haggerty C. C., Caprioli D., 2019, ApJ, 887, 165

Hirashita H., Inoue A. K., 2019, MNRAS, 487, 961

Hirashita H., Lin C.-Y., 2020, Planetary Space Sci., 183, 104504

Holcomb C., Spitkovsky A., 2019, ApJ, 882, 3

Hopkins P. F., Squire J., 2018, MNRAS, 479, 4681 (HS18)

Hopkins P. F., Squire J., Seligman D., 2020c, MNRAS, 496, 2123

Hopkins P. F. et al., 2020d, MNRAS, 492, 3465

Hopkins P. F. et al., 2021a, MNRAS, 501, 3663

Hopkins P. F., Squire J., Chan T. K., Quataert E., Ji S., Kereš D., FaucherGiguère C.-A., 2021b, MNRAS, 501, $4184(\mathrm{H}+20)$

Ishibashi W., Fabian A. C., 2015, MNRAS, 451, 93

Ji S. et al., 2020, MNRAS, 496, 4221

Jokipii J. R., 1966, ApJ, 146, 480

Krapp L., Benítez-Llambay P., Gressel O., Pessah M. E., 2019, ApJ, 878, L30

Kulsrud R., Pearce W. P., 1969, ApJ, 156, 445

Lacki B. C., Thompson T. A., Quataert E., Loeb A., Waxman E., 2011, ApJ, 734,107

Lazarian A., 2016, ApJ, 833, 131

Lazarian A., Yan H., 2002, ApJ, 566, L105

Lee M. A., Völk H. J., 1973, Astrophys. Space. Sci., 24, 31

Lee H., Hopkins P. F., Squire J., 2017, MNRAS, 469, 3532

Lopez L. A. et al., 2018, ApJ, 867, 44

Mathis J. S., Rumpl W., Nordsieck K. H., 1977, ApJ, 217, 425

Maurin D., Putze A., Derome L., 2010, A\&A, 516, A67

McKenzie J. F., Voelk H. J., 1982, A\&A, 116, 191

Ménard B., Scranton R., Fukugita M., Richards G., 2010, MNRAS, 405, 1025

Micelotta E. R., Matsuura M., Sarangi A., 2018, Space Sci. Rev., 214, 53

Moseley E. R., Squire J., Hopkins P. F., 2019, MNRAS, 489, 325

Murray N., Quataert E., Thompson T. A., 2005, ApJ, 618, 569

Peek J. E. G., Ménard B., Corrales L., 2015, ApJ, 813, 7

Peters T. et al., 2017, MNRAS, 467, 4322

Pfrommer C., Pakmor R., Simpson C. M., Springel V., 2017, ApJ, 847, L13

Schekochihin A. A. et al., 2009, ApJ Supp., 182, 310

Seligman D., Hopkins P. F., Squire J., 2019, MNRAS, 485, 3991

Shukla P. K., Mamun A. A., 2002, Introduction to Dusty Plasma Physics. IOP Publishing, Bristol and Philadelphia

Silsbee K., Ivlev A. V., Gong M., 2020, preprint (arXiv:2011.13879)

Skilling J., 1971, ApJ, 170, 265

Skilling J., 1975a, MNRAS, 172, 557

Skilling J., 1975b, MNRAS, 173, 245

Squire J., Hopkins P. F., 2018, ApJ, 856, L15

Squire J., Quataert E., Schekochihin A. A., 2016, ApJ, 830, L25

Squire J., Schekochihin A. A., Quataert E., 2017, New J. Phys., 19, 055005

Strong A. W., Moskalenko I. V., 1998, ApJ, 509, 212

Su K.-Y. et al., 2020, MNRAS, 491, 1190

Thomas T., Pfrommer C., 2019, MNRAS, 485, 2977
Tielens A. G. G. M., 2005, The Physics and Chemistry of the Interstellar Medium. Cambridge Univ. Press, Cambridge

Tripathi K. D., Sharma S. K., 1996, Phys. Plasmas, 3, 4380

Tumlinson J., Peeples M. S., Werk J. K., 2017, A\&A, 55, 389

Völk H. J., Cesarsky C. J., 1982, Z. Naturforsch., 37, 809

Weidl M. S., Winske D., Niemann C., 2019, ApJ, 873, 57

Weingartner J. C., 2004, in Witt A. N., Clayton G. C., Draine B. T., eds, ASP Conf. Ser. Vol. 309, Astrophysics of Dust. Astron. Soc. Pac., San Francisco, p. 453

Weingartner J. C., Draine B. T., 1974, ARA\&A, 12, 71

Weingartner J. C., Draine B. T., 2001a, ApJ, 548, 296

Weingartner J. C., Draine B. T., 2001b, ApJ, 553, 581

Weingartner J. C., Draine B. T., 2001c, ApJS, 134, 263

Wentzel D. G., 1969, ApJ, 156, 303

Wiener J., Zweibel E. G., Oh S. P., 2018, MNRAS, 473, 3095

Yan H., Lazarian A., 2002, Phys. Rev. Lett., 89, 281102

Yan H., Lazarian A., 2004, ApJ, 614, 757

Yan H., Lazarian A., 2008, ApJ, 673, 942

Yan H., Lazarian A., Draine B. T., 2004, ApJ, 616, 895

Zubko V., Dwek E., Arendt R. G., 2004, ApJS, 152, 211

Zweibel E. G., 2017, Phys. Plasmas, 24, 055402

\section{APPENDIX A: GROWTH AND DAMPING WITH A SPECTRUM OF GRAIN SIZES}

In this appendix, we detail the derivations of equations (8) and (9), explore some of the properties of these, and discuss some limits on their validity. We first discuss some technical features of the discrete growth rate calculation in Section A1, then how to take the continuum limit in Section A2. The possible influence of a dust velocity dispersion induced by turbulence is considered in Appendix A3. Although the general procedure - computing a discrete growth rate first, then taking the limit $N_{d} \rightarrow \infty$ - may seem more complex than starting directly from equations for the continuum limit of equations (3) to (6), the method seems to have some important advantages. First, in similar calculations of dust interacting with an ion-electron plasma only through electromagnetic fields (i.e. without drag), but taking the limit $N_{d} \rightarrow \infty$ before computing a dispersion relation, Tripathi \& Sharma (1996), Cramer et al. (2002) find expressions for the growth rate that can be evaluated analytically only for specific powerlaw dust-density distributions. Secondly, our calculation provides useful physical intuition on its limits at finite dust stopping time $t_{s}$, explaining why the result is effectively independent of $t_{s}$ in the regimes of interest.

\section{A1 Discrete distribution}

As discussed in Section 3.2, the growth rate of a discrete collection of grains can be straightforwardly obtained by computing the dispersion relation from the linearization of equations (3) to (6). Assuming that the solution of the resulting polynomial is of the form $\omega=k v_{A}+$ $\omega^{(1)}$, where $\omega^{(1)} \sim \mathcal{O}(\mu)$, then expanding in $\mu \ll 1$, assuming all individual dust densities $\mu_{i}$ scale with $\mu$, leads to

$$
\begin{aligned}
& \mu\left(-\omega^{(1)}+\sum_{i=1}^{N_{d}} \mu_{i} \omega_{A} \mathcal{W}_{s, i} \frac{t_{L, i}^{2}\left(1+i \omega_{A} t_{s, i} \mathcal{W}_{s, i}\right)-t_{s, i}^{2} \mathcal{W}_{s, i}}{t_{s, i}^{2}+t_{L, i}^{2}\left(1+i \omega_{A} t_{s, i} \mathcal{W}_{s, i}\right)^{2}}\right) \\
& \quad \times \prod_{i=1}^{N_{d}} \mathcal{W}_{s, i}\left(1+i \omega_{A} t_{s, i} \mathcal{W}_{s, i}\right)+\mathcal{O}\left(\mu^{2}\right)=0
\end{aligned}
$$

Solution of the first term in the product gives equation (8), after normalizing the wavenumber of the mode to the resonant wavenumber using $\omega_{A}=k v_{A}=\left(t_{L} \mathcal{W}_{s, i} \bar{\lambda}_{i}\right)^{-1}$, where $\bar{\lambda}_{i} \lambda_{\text {res }, i}=k^{-1}$ and $\lambda_{\text {res }, i} \equiv v_{A} t_{L, i} \mathcal{W}_{s, i}$. 
Equation (A1) is valid when $\mu_{i}$ is sufficiently small such that the effect of the dust gyro-motion can be considered a small perturbation to the Alfvén wave. At larger $\mu_{i}$, the structure of the linear system is modified because the matrix becomes defective (nondiagonalizable), causing the growth rate to scale with $\sim \mu_{i}^{1 / 2}$ rather than the assumed $\sim \mu_{i}$, and thus invalidating the expression (A1). As discussed in Squire \& Hopkins (2018) (see discussion below their equation 12), mathematically this implies that the growth rate of the mode must be less than that of the 'resonant drag instability' between the dust gyro-motion and the Alfvén wave, which has the growth rate,

$\Im(\omega) \approx \frac{1}{2} \mu_{i}^{1 / 2}\left|t_{L, i}\right|^{-1}\left|\mathcal{W}_{s, i}\right|^{1 / 2}=\left.\frac{1}{2} \mu_{i}^{1 / 2}\left|\mathcal{W}_{s, i}\right|^{3 / 2} \omega_{A}\right|_{k=\lambda_{\text {res }, i}^{-1}}$

at resonance $\left(k=\lambda_{\text {res, } i}^{-1}\right)$ for a single grain population $i$ (HS18). In other words, equation (A2), rather than equation (8), is the correct expression at larger $\mu_{i}$. Equations (A1) and (8) are thus valid if $\left.\tilde{\omega}_{i}^{(1)}\right|_{\bar{\lambda}_{i}=1} \lesssim \mu_{i}^{-1 / 2} \mathcal{W}_{s, i}^{3 / 2}$, or - expanding $\tilde{\omega}_{i}^{(1)}$ in $\bar{t}_{s, i} \gg 1$ to find $\left.\tilde{\omega}_{i}^{(1)}\right|_{\bar{\lambda}_{i}=1} \approx \mathcal{W}_{s, i}^{3} \bar{t}_{s, i} / 2+\mathcal{O}\left(\bar{t}_{s, i}^{-1}\right)-$ for

$\left|\mathcal{W}_{s, i}\right|^{3 / 2} \bar{t}_{s, i} \lesssim \mu_{i}^{-1 / 2}$

This condition compares well against full numerical solutions to the dispersion relation.

\section{A2 Continuum limit}

For $\bar{t}_{s, i} \gg 1$, the contribution to growth or damping from a single grain species (the function $\tilde{\omega}_{i}^{(1)}$ in the sum of equation 8), becomes very sharply peaked around $\bar{\lambda}_{i}=1$. For illustration, we plot $\tilde{\omega}_{i}^{(1)}\left(\bar{\lambda}_{i}\right)$ in the top panel of Fig. A1, showing (i) how its peak value (at $\bar{\lambda}_{i}=1$ ) scales linearly with $\bar{t}_{s, i}\left[\tilde{\omega}_{i}^{(1)}(1) \approx \mathcal{W}_{s, i}^{3} \bar{t}_{s, i} / 2+\mathcal{O}\left(\bar{t}_{s, i}^{-1}\right)\right]$, (ii) that $\tilde{\omega}_{i}^{(1)} \rightarrow 0$ as $\bar{\lambda}_{i} \rightarrow \infty$, and (iii) that $\tilde{\omega}_{i}^{(1)}(0) \approx-\bar{t}_{s, i}^{-1}+\mathcal{O}\left(\bar{t}_{s, i}^{-3}\right)$. Moreover, it transpires that

$\int_{0}^{\infty} \mathrm{d} \bar{\lambda}_{i} \tilde{\omega}_{i}^{(1)}=\frac{\pi}{2} \operatorname{sgn}\left(\mathcal{W}_{s, i}\right) \mathcal{W}_{s, i}^{2}$

implying that at $\bar{t}_{s, i} \gg 1, \tilde{\omega}_{i}^{(1)}$ is well approximated by a delta function, with no $\bar{t}_{s, i}$ dependence: $\tilde{\omega}_{i}^{(1)} \approx(\pi / 2) \operatorname{sgn}\left(\mathcal{W}_{s, i}\right) \mathcal{W}_{s, i}^{2} \delta\left(\bar{\lambda}_{i}-\right.$ $1)=(\pi / 2) \operatorname{sgn}\left(\mathcal{W}_{s, i}\right) \mathcal{W}_{s, i}^{2} \lambda_{\text {res }, i} \delta\left(k^{-1}-\lambda_{\text {res }, i}\right)$. Given that the total growth rate from $N_{d}$ species is simply the sum of the individual growth rates, we can derive an expression for the continuum damping/growth rate, $\gamma(k)$, by converting the sum in equation (8) to an integral. This is done by stipulating that the area under $\gamma(k)$ between resonances, $\gamma(k) d \lambda_{\text {res, } i}=\gamma(k)\left(\lambda_{\text {res }, i+1}-\lambda_{\text {res, } i}\right)$ is equal to the area under the discrete growth rate at the same resonance, which is $\Im\left[\omega^{(1)}\left(\lambda_{\text {res }, i}\right)\right] \mathrm{d} \lambda_{\text {res }, i} \approx \mu_{i}(\pi / 2) \omega_{A} \operatorname{sgn}\left(\mathcal{W}_{s, i}\right) \mathcal{W}_{s, i}^{2} \lambda_{\text {res }, i}$, where in the final step we have used the delta-function approximation for $\tilde{\omega}_{i}^{(1)}$. Replacing $\mu_{i}$ by $d \mu$ with $\mu_{0}=\sum_{i=1}^{N_{d}} \mu_{i}=\int \mathrm{d} \mu$, we get

$$
\begin{aligned}
\gamma(k) & =\frac{\Im\left[\omega^{(1)}\left(\lambda_{\text {res }, i}\right)\right] \mathrm{d} \lambda_{\text {res }, i}}{\mathrm{~d} \lambda_{\text {res }, i}} \\
& =\omega_{A} \frac{\pi}{2} \operatorname{sgn}\left(\mathcal{W}_{s}\right) \frac{\mathrm{d} \mu}{\mathrm{d} \ln a_{d}}\left(\frac{\mathrm{d} \lambda_{\text {res }}}{\mathrm{d} \ln a_{d}}\right)^{-1} \mathcal{W}_{s}^{2}\left(a_{d}\right) \lambda_{\text {res }}\left(a_{d}\right) \\
& =\omega_{A} \frac{\pi}{2} \operatorname{sgn}\left(\mathcal{W}_{s}\right) \frac{\mathrm{d} \mu}{\mathrm{d} \ln a_{d}}\left(\frac{\mathrm{d} \ln \left|t_{L}\right|}{\mathrm{d} \ln a_{d}}+\frac{\mathrm{d} \ln \left|\mathcal{W}_{s}\right|}{\mathrm{d} \ln a_{d}}\right)^{-1} \mathcal{W}_{s}^{2},
\end{aligned}
$$

where all functions of $a\left(t_{L}\left(a_{d}\right), \mathcal{W}_{s}\left(a_{d}\right)\right.$ etc.) are converted to functions of $k$ by inverting $k^{-1}=\lambda_{\text {res }}\left(a_{d}\right)=v_{A} t_{L} \mathcal{W}_{s}$ (note that
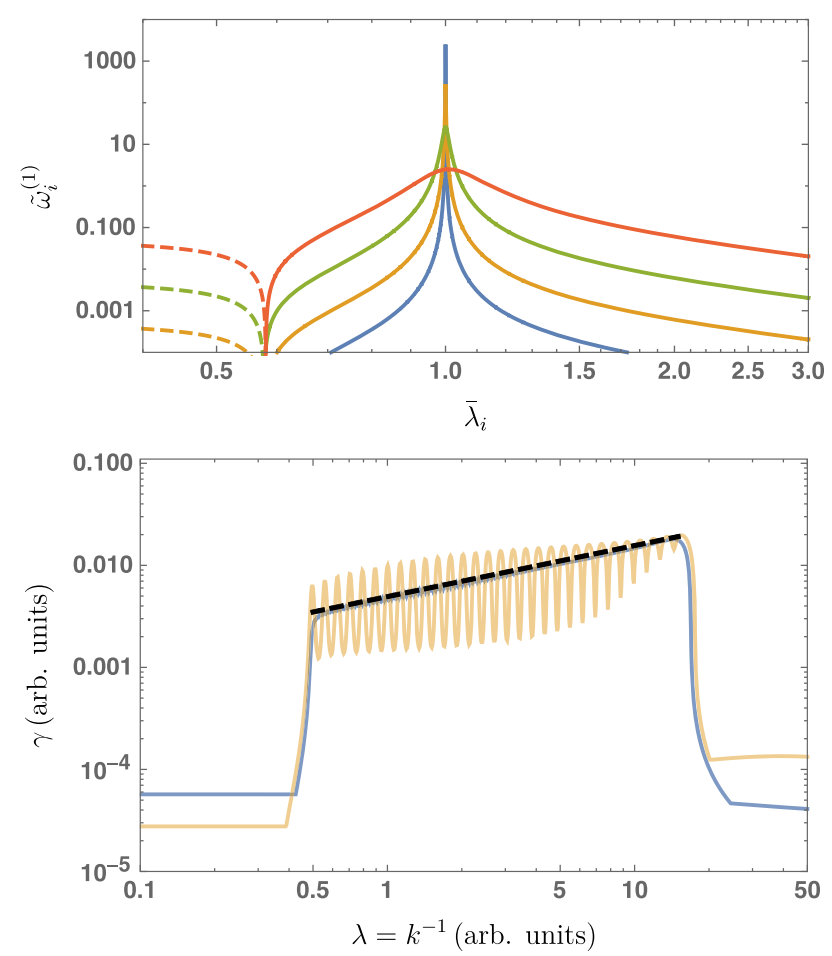

Figure A1. Top panel: single-grain contribution to the growth rate $\tilde{\omega}_{i}^{(1)}$ (equation 8) for $\mathcal{W}_{s, i}=1$, with $\bar{t}_{s, i}=t_{s, i} \omega_{A}$ in the range $\bar{t}_{s, i}=$ $\left\{10^{4}, 10^{3}, 10^{2}, 10^{1}\right\}$ (blue, orange, green, and red curves, respectively). The dashed lines show where $\tilde{\omega}_{i}^{(1)}<0\left(\tilde{\omega}_{i}^{(1)} \approx \bar{t}_{s, i}^{-1}\right.$ for $\left.\bar{t}_{s, i} \gg 1\right)$. Bottom panel: comparison of the continuum approximation equation (9) (black dashed line) to the full dispersion relation computed numerically from the linearization of equations (2) to (6). The blue line shows the dispersion relation with 150 dust species, while the light-orange line shows the case with 30 dust species, illustrating the convergence towards the continuum solution. We use arbitrary units with $a_{d}$ ranging from $a_{d}=1$ to $a_{d}=30$ in logarithmically spaced increments, $v_{A}=1, \beta=10, t_{L}=0.5 a_{d}^{0.5}, \mathcal{W}_{s}=a_{d}^{0.5}, t_{s}=30 a_{d}$, a mass distribution $\mathrm{d} \bar{\mu} / \mathrm{d} \ln a_{d} \propto a_{d}^{0.5}$, and a total dust density $\mu_{0}=0.01$. The analytic expression for the growth rate follows from inverting the resonance condition, $v_{A} t_{L} \mathcal{W}_{s}=k^{-1}$, to give $a_{d}=2 / k$, which gives $\gamma \approx 0.5 \mu_{0} k^{-1 / 2}$ using equation (9).

$k^{-1}$ can be negative, which allows for different mode polarizations depending on the sign of $t_{L}$ and $\mathcal{W}_{s}$ ).

To understand possible restrictions on equation (A5), let us note two key points that are implicit in the derivation. First, as we approach the true continuum limit, the condition equation (A3) must become satisfied at some point, since we are taking the limit as $\mu_{i} \rightarrow 0$. Secondly, although we used the delta function approximation to $\tilde{\omega}_{i}^{(1)}$ to derive equation (A5), it is clear that the final answer will be identical if the resonances overlap (finite $\bar{t}_{s}$ ): each of the nearby resonances from different sized grains will contribute a small amount to the region $\mathrm{d} \lambda_{\text {res, } i}$, whose total - assuming that the peak of $\tilde{\omega}_{i}^{(1)}$ does not vary much across the width of the contribution from a single grain - will add up to the same as the delta function approximation. We thus see that the effect of finite $\bar{t}_{s}$ is to smooth out any sharp features in the continuum growth rate expression equation (9) or equation (A5) by the width of an individual resonance, which is $\mathrm{d} \bar{\lambda}_{i} \sim \bar{t}_{s, i}^{-1}$ or

$\mathrm{d} k \sim \frac{k^{2} \lambda_{\text {res }}}{\omega_{A} t_{s}}=k\left|\mathcal{W}_{s}\right| \frac{\left|t_{L}\right|}{t_{s}}$ 
In addition, minor (order $\bar{t}_{s}^{-1}$ ) inaccuracies arise due to $\tilde{\omega}_{i}^{(1)}$ becoming negative as $k \rightarrow \infty$.

We compare the continuum approximation equation (9) to numerical calculations of the full dust-gas dispersion relation in the bottom panel of Fig. A1. Parameters $t_{s}\left(a_{d}\right), t_{L}\left(a_{d}\right)$, and $\mathcal{W}_{s}\left(a_{d}\right)$ are chosen as arbitrary functions for illustrative purposes. The comparison of the orange and blue curves, which are the numerical calculations with 30 and 150 dust species, respectively, illustrates the accuracy of the continuum approximation (black dashed line) in the region of resonances once the individual resonances overlap (blue line). The continuum approximation predicts no growth or damping for wavenumbers outside of the band of resonances, which we see is not completely accurate (although growth rates are $\sim 10^{-2}$ lower outside the band).

\section{A3 Dust velocity dispersion}

It is expected that even if $w_{s}=0$, the dust should have some nonzero velocity dispersion due to the effects of turbulent gas motions (Lazarian \& Yan 2002; Lee, Hopkins \& Squire 2017), likely with the dispersion of perpendicular velocities dominating over that of parallel velocities for most magnetized turbulence (Yan et al. 2004). Including this formally and in detail requires recourse to a fundamentally kinetic description of the dust; this would involve linearizing a dust Vlasov equation for its distribution function $f_{d}\left(\boldsymbol{x}, \boldsymbol{v}, a_{d}\right)$, which is beyond the scope of this work. However, in order to get a basic idea of whether such effects are likely to be important, we can consider adding in a dust pressure term into the dust velocity evolution equation (5). This must have the form $\nabla_{d \perp} P_{d \perp, i}+\partial_{z} P_{d \|, i} \hat{z}$, where $\nabla_{\perp}$ is the gradient in the perpendicular ( $x$ and $y$ ) direction and

$P_{d \perp, i}$ and $P_{d \|, i}$ refer to the dust velocity dispersions perpendicular and parallel to the field, respectively (the equilibrium off-diagonal components of the dispersion tensor must be small if the dust velocity distribution is symmetric around the magnetic field). While $P_{d \perp, i}$ and $P_{d \|, i}$ are not determined without recourse back to the kinetic description, a simple isothermal closure $-P_{d \perp, i}=\rho_{d, i} c_{d \perp 0, i}^{2}$ and $P_{d \|, i}=\rho_{d, i} c_{d \| 0, i}^{2}$, where $c_{d \perp 0, i}$ and $c_{d \| 0, i}$ are fixed - should at least give an idea of whether the velocity dispersion should fundamentally change the modes of interest. Repeating the calculation above, one finds that it does not: the perpendicular pressure has no effect whatsoever, because it depends only on perpendicular gradients and we explicitly focus only on parallel modes; the parallel pressure modifies the final part of equation (A1), changing the term in the product to $\mathcal{W}_{s, i}\left(1+i \omega_{A} t_{s, i} \mathcal{W}_{s, i}\right)-i k_{z}^{2} t_{s, i} c_{d \| 0, i}^{2} / \omega_{A}$, but this has no effect on the modes of interest either (at small $\mu_{i}$ ). This illustrates that the direct effect of dust pressure is unimportant for the Alfvénic modes of interest, although it does not capture more complex kinetic effects such as the broadening of the distribution of resonances due to the range of parallel velocities. The mode's indifference to the perpendicular velocity distribution is straightforward to understand physically: the dust's gyro-time, which determines the damping through the resonance with the Alfvén wave $\left(\bar{\lambda}_{i}=1\right)$, is independent of its perpendicular velocity.

This paper has been typeset from a $\mathrm{T}_{\mathrm{E}} \mathrm{X} / \mathrm{LT} \mathrm{E} \mathrm{X}$ file prepared by the author. 